\title{
Regularization modeling for large-eddy simulation of homogeneous isotropic decaying turbulence
}

\author{
Bernard J Geurts ${ }^{1,2,6}$, Arkadiusz K Kuczaj ${ }^{3}$ and Edriss S Titi ${ }^{4,5}$ \\ ${ }^{1}$ Multiscale Modeling and Simulation, J.M. Burgers Center, AACS, Faculty EEMCS, \\ University of Twente, PO Box 217, 7500 AE Enschede, The Netherlands \\ ${ }^{2}$ Anisotropic Turbulence, Fluid Dynamics Laboratory, Department of Applied Physics, \\ Eindhoven University of Technology, PO Box 513, 5600 MB Eindhoven, The Netherlands \\ ${ }^{3}$ Computational Fluid Dynamics, Nuclear Research and Consultancy Group (NRG), PO Box 25, \\ 1755 ZG Petten, The Netherlands \\ ${ }^{4}$ Department of Mathematics and Department of Mechanical and Aerospace Engineering, \\ University of California, Irvine, CA 92697-3875, USA \\ ${ }^{5}$ Department of Computer Science and Applied Mathematics, Weizmann Institute of Science, \\ Rehovot 76100, Israel \\ E-mail: b.j.geurts@utwente.nl
}

Received 20 December 2007, in final form 13 April 2008

Published DD MMM 2008

Online at stacks.iop.org/JPhysA/41/000000

\begin{abstract}
Inviscid regularization modeling of turbulent flow is investigated. Homogeneous, isotropic, decaying turbulence is simulated at a range of filter widths. A coarse-graining of turbulent flow arises from the direct regularization of the convective nonlinearity in the Navier-Stokes equations. The regularization is translated into its corresponding sub-filter model to close the equations for large-eddy simulation (LES). The accuracy with which primary turbulent flow features are captured by this modeling is investigated for the Leray regularization, the Navier-Stokes- $\alpha$ formulation (NS- $\alpha$ ), the simplified Bardina model and a modified Leray approach. On a PDE level, each regularization principle is known to possess a unique, strong solution with known regularity properties. When used as turbulence closure for numerical simulations, significant differences between these models are observed. Through a comparison with direct numerical simulation (DNS) results, a detailed assessment of these regularization principles is made. The regularization models retain much of the small-scale variability in the solution. The smaller resolved scales are dominated by the specific sub-filter model adopted. We find that the Leray model is in general closest to the filtered DNS results, the modified Leray model is found least accurate and the simplified Bardina and NS- $\alpha$ models are in between, as far as accuracy is concerned. This rough ordering is based on the energy decay, the Taylor Reynolds number and the velocity skewness, and on detailed characteristics of the energy dynamics, including spectra of the energy, the energy transfer and the transfer power. At
\end{abstract}

6 International Collaboration for Turbulence Research (ICTR). 
filter widths up to about $10 \%$ of the computational domain-size, the Leray and NS- $\alpha$ predictions were found to correlate well with the filtered DNS data. Each of the regularization models underestimates the energy decay rate and overestimates the tail of the energy spectrum. The correspondence with unfiltered DNS spectra was observed often to be closer than with filtered DNS for several of the regularization models.

PACS numbers: 47.27.ep, 47.27.Gs

Mathematics Subject Classification: 76F05, 76F65, 81T80

This paper is dedicated to Darryl D Holm, on the occasion of his 60th birthday.

(Some figures in this article are in colour only in the electronic version)

\section{Introduction}

Turbulence arises in a variety of flows in nature and technology. Although as a rule a strict definition is not provided in the literature, turbulence is associated with the presence of a broad dynamic range of the length- and time-scales in a flow [1,2]. Through nonlinear interactions among these scales, a complex multiscale dynamics results [3]. For incompressible fluids, such as water or air at low speeds, this is governed by the Navier-Stokes equations. Under realistic flow conditions, the range of relevant dynamical scales is too large to be explicitly and completely resolved numerically. A pragmatic answer to cope with this situation is obtained by restricting computational models to resolving only the primary features of the flow $[4,5]$. The latter requires considerably reduced computational effort and hence these simulations at least become feasible. However, the main issue is not about mere feasibility, but whether or not the primary flow features can be captured accurately when such a coarsened flow description is considered.

In large-eddy simulation, a coarsened representation of a turbulent flow is formulated based on the application of an explicit spatial filter to the Navier-Stokes equations [6]. Such an explicit filtering introduces the filter width $\Delta$ as a new length-scale, which provides external control over the effective dynamic range of the smoothed flow. At the same time, the filtering of the convective nonlinearity introduces an explicit closure problem. As witnessed in the LES literature, this closure problem has triggered considerable inventiveness in the construction of the so-called sub-filter models, which approximate the dynamic consequences of the smallscales for the evolution of the resolved large scales. Much work has been devoted to assessing such sub-filter models, many of which are loosely motivated by an argumentation based on statistical properties of a turbulent flow. Good examples are eddy-viscosity models such as Smagorinsky's model [7], or scale-similarity models such as Bardina's model [8, 9]. An alternative route to sub-filter modeling is achieved by adhering to the transport structure of the filtered Navier-Stokes equations. Rather than closing the filtered equations by introducing a dissipative flux term, the nature of the closure problem can be used as a point of departure [10]. An important class of sub-filter models obtained in this way is the 'regularization' model. In these models the filtered convective fluxes in the Navier-Stokes equations are directly modified, thereby retaining much of the mathematical properties of the filtered equations. A recent example of such an approach to sub-filter modeling was motivated by the seminal paper by Leray [11] and applied to turbulent mixing in [12, 13] and turbulence closure [14]. 
In this paper we assess the quality of sub-filter models obtained on the basis of regularization principles. We include the Leray principle [11, 12, 14], the NS- $\alpha$ formulation [15-20], the simplified Bardina model [21-23] and a recent proposal based on a modified Leray principle [24]. These inviscid, i.e., non-hyper-viscous, regularization principles are appealing as the corresponding system of nonlinear partial differential equations is known to possess a unique solution with known regularity [11, 14, 17, 18, 21-24]. These models were shown to predict large scales of ensemble averages of turbulent flows in channels and pipes at very large Reynolds numbers in close agreement with experimental data $[15,16,25]$ (see also [26] for the Clark- $\alpha$ model). However, that by itself is no guarantee for accurate capturing of the primary turbulent flow features in actual large-eddy simulation models. Therefore, we investigate these regularization principles in the large-eddy simulations of homogeneous, isotropic, decaying turbulence. Comparison with data from a direct numerical simulation of the Navier-Stokes equations provides a conclusive opportunity for assessing the suitability of a regularization principle as the 'generator' of a sub-filter model. We will show that remarkably large differences occur between flow predictions obtained on the basis of the above regularization principles, despite the fact that these formulations share a common perspective on the smoothing of small scales. We analyze this on the basis of a large number of large-eddy simulations in which the spatial resolution $N=1 / h$, with $h$ the mesh-spacing, and the filter width $\Delta$ were systematically varied.

The purpose of this paper is to investigate to what extent the global regularity of solutions to certain mollifications/regularizations of the three-dimensional Navier-Stokes equations is related to the accurate capturing of the spatially filtered flow representation. The effect of the regularization models on the large energetic scales in a turbulent flow is quite limited. However, their action on the small-scale features in a flow differs considerably, particularly at large filter widths. While this combines into a quite similar capturing of mean flow properties, fluctuating properties and the spectral composition of the grid-independent numerical solution are found to differ considerably. We compare the four regularization models, mentioned above, with filtered DNS data for homogeneous, isotropic, decaying turbulence. Sufficiently small scales in turbulent flows are often postulated to possess 'universal properties'. In particular, it is assumed that such small scales display dynamical behavior that is quite independent of the large-scale flow that carries these small scales. This motivates the study of homogeneous, isotropic, decaying turbulence as a canonical flow-type in which explicit sub-filter models should be tested. We consider this flow at a variety of spatial resolutions and filter widths to investigate the flow predictions under the conditions of marginal resolution as well as in the case of approximate grid-independence.

The organization of this paper is as follows. In section 2, we introduce the direct regularization modeling of turbulence in the context of the filtering approach to large-eddy simulation. Section 3 is devoted to a description of the numerical method and the simulation of homogeneous, isotropic, decaying turbulence. Statistical properties of regularized turbulence are presented in section 4 , while a detailed discussion of the energy dynamics is provided in section 5. Concluding remarks are reported in section 6.

\section{Regularization modeling of turbulence}

In this section, we review the filtering approach to large-eddy simulation and connect this to regularization modeling. We present the regularized Navier-Stokes equations in detail in case the Leray, the NS- $\alpha$, the simplified Bardina and the modified Leray regularizations are combined with explicit Helmholtz filtering, i.e., a spatial convolution with a Bessel potential [27], which is the Green function of the Helmholtz operator. 
The flow of a constant density fluid such as water is governed by the Navier-Stokes equations subject to the constraint of incompressibility. Mathematically, the conservation of mass and momentum can be expressed as

$$
\begin{aligned}
& \partial_{j} u_{j}=0, \\
& \partial_{t} u_{i}+\partial_{j}\left(u_{j} u_{i}\right)+\partial_{i} p-\frac{1}{R e} \partial_{j j} u_{i}=0, \quad i=1,2,3,
\end{aligned}
$$

where $u_{i}$ denotes the velocity in the $x_{i}$ direction and $p$ is the pressure. Partial derivatives with respect to the time $t$ and spatial coordinate $x_{i}$ are denoted by $\partial_{t}$ and $\partial_{i}$, respectively. The Reynolds number $R e$ measures the relative importance of the convective flux $\partial_{j}\left(u_{j} u_{i}\right)$ and the viscous flux $\partial_{j j} u_{i}$. It is defined as $R e=\left(U_{r} \ell_{r}\right) / v$, where $U_{r}, \ell_{r}$ and $v$ denote the reference velocity, length-scale and kinematic viscosity, respectively, that were adopted to nondimensionalize the equations.

The solution to (1) and (2) has a dynamic range that extends to the flow features of the order of the Kolmogorov dissipation scale $\eta$ [29, 30]. For homogeneous, isotropic turbulence, $\eta \sim R e^{-3 / 4}$. This scaling law implies that flows at high Reynolds numbers require very small mesh-spacings $h \lesssim O(\eta)$ in order to be properly resolved numerically. In realistic cases, the Reynolds number is so high that this requirement cannot be met with currently available computing infrastructure and resources. As an example, Reynolds numbers as high as $R e=10^{8}$ are associated with flows in the atmosphere, while $R e=10^{6}-10^{7}$ arises in the most applications of commercial aircraft. Even larger Reynolds numbers are connected to flow in the ocean and in relation to the evolution of structures in the universe.

Various applications or scientific questions do not require accurate predictions of all dynamic scales up to $\eta$. In fact, most of the energy contained in a flow is represented by its primary flow features. For various problems, it suffices to develop computational models that capture the larger scales in a flow only. How this general observation should be translated into a specific computational modeling is a matter of ongoing research for which only rather crude suggestions have been made. As a general 'rule of thumb', proposed by Pope [1], capturing of about $80 \%$ of the total kinetic energy should yield a sufficiently accurate description of mean flow profiles. Alternatively, it was found that filter widths up to about $5 \%$ of a typical large scale of the flow field, yield a good correspondence with filtered DNS findings [15, 16, 19, 31, 32]. In doing so, one may take the liberty to sacrifice reliable predictions of the smallest scales, provided there is no 'contamination' of such small-scale errors toward the larger scales. This is a central premise of coarsened flow descriptions [4].

An external control over the dynamic range of the computational model can be obtained by the spatial filtering of (1) and (2). This is the basis of the filtering approach to large-eddy simulation [6]. As a first step, one introduces a convolution filter $L$. In one spatial dimension this is conveniently expressed as

$$
\bar{u}(x, t)=L(u)=\int_{-\infty}^{\infty} G(x-\xi) u(\xi, t) \mathrm{d} \xi,
$$

where $G$ denotes the filter-kernel and $\bar{u}$ the filtered solution obtained from the unfiltered solution $u$. This kernel is assumed to be characterized by a filter width $\Delta$, e.g., defined in terms of its second moment [33]. Various filter-kernels have been suggested in the literature. In this paper, we will adopt the Helmholtz filter, based on the Bessel potential kernel $[15-19,27]$. This filter is defined as $L=H^{-1}$, where the Helmholtz operator $H$ is given by

$$
u=H(\bar{u})=\left(1-\alpha^{2} \partial_{x x}\right) \bar{u} .
$$

Here we introduced the 'Helmholtz-length' $\alpha$, which defines the effective width of this filter. Comparing (4) to Taylor expansions of the effect of popular second-order filters, such as 
the top-hat and the Gaussian filters, it was shown in [28] that $\alpha^{2} \approx \Delta^{2} / 24$. Thus, the Helmholtz-length is about $1 / 5$ of the filter width of second-order filters.

The action of the Helmholtz filter on $u=\mathrm{e}^{2 \pi \mathrm{i} k x}$, with $\mathrm{i}^{2}=-1$ and $k \in \mathbb{Z}$, can be written as $\bar{u}=L(u)=\mathcal{H}(k \alpha) \mathrm{e}^{2 \pi \mathrm{i} k x}$, where the Fourier transform of the Helmholtz kernel is given by

$$
\mathcal{H}(k \alpha)=\frac{1}{1+4 \pi^{2}(k \alpha)^{2}} .
$$

Correspondingly, the application of the Helmholtz filter to an arbitrary solution $u$ can be expressed most concisely in terms of its effect on the Fourier series expansion of $u$. Specifically, with $u$ represented by Fourier coefficients $\left\{c_{k}\right\}$ we may write

$$
\bar{u}(x, t)=H^{-1}\left(\sum_{k=-\infty}^{\infty} c_{k} \mathrm{e}^{2 \pi \mathrm{i} k x}\right)=\sum_{k=-\infty}^{\infty} \mathcal{H}(k \alpha) c_{k} \mathrm{e}^{2 \pi \mathrm{i} k x} .
$$

This illustrates the attenuation of each individual Fourier component as a result of Helmholtz filtering. Scales for which $k \alpha \gg 1$ are effectively removed from the solution. Specifically, if we define the 'effective' cut-off at $\mathcal{H}(k \alpha)=1 / 2$ [34], then solution-components with $k \alpha \leqslant 1 /(2 \pi)$ can be interpreted as 'resolved' scales, while the smaller scales are 'sub-filter' scales. In three dimensions we adopt the Helmholtz filter with

$$
\mathcal{H}(k \alpha)=\frac{1}{1+4 \pi^{2} \alpha^{2}\left(k_{1}^{2}+k_{2}^{2}+k_{3}^{2}\right)},
$$

where $\mathbf{k}=\left[k_{1}, k_{2}, k_{3}\right]$ is the vector of wavenumbers.

The application of any convolution filter to the governing equations (1) and (2) can be expressed in terms of the 'LES template' as

$$
\begin{aligned}
& \partial_{j} \bar{u}_{j}=0, \\
& \partial_{t} \bar{u}_{i}+\partial_{j}\left(\bar{u}_{j} \bar{u}_{i}\right)+\partial_{i} \bar{p}-\frac{1}{R e} \partial_{j j} \bar{u}_{i}=-\partial_{j} \tau_{i j},
\end{aligned}
$$

where we observe the Navier-Stokes equations on the left-hand side in terms of the filtered velocity and pressure, and the divergence of the so-called turbulent stress tensor $\tau_{i j}$ on the right-hand side. The turbulent stress tensor is given by

$$
\tau_{i j}=\overline{u_{j} u_{i}}-\bar{u}_{j} \bar{u}_{i}=L\left(u_{j} u_{i}\right)-L\left(u_{j}\right) L\left(u_{i}\right)=\left[L, \Pi_{i j}\right](\mathbf{u}) .
$$

This tensor arises as a commutator of the filter operation and the quadratic nonlinearity in the convective fluxes, where $[A, B]=A \circ B-B \circ A$ and $\Pi_{i j}(\mathbf{u})=u_{i} u_{j}$. The commutator properties of $\tau_{i j}$ are in a number of ways similar to those of the Poisson bracket in classical mechanics, which provides the basis for dynamic sub-filter modeling in which model parameters follow from a solution-adaptive least-squares optimization [35, 36].

The filtered equations are not closed as the evaluation of the turbulent stress tensor cannot be performed on the basis of the filtered solution alone. The central challenge in large-eddy modeling is to approximate $\partial_{j} \tau_{i j}$ in terms of a model operating on $\overline{\mathbf{u}}$. Various proposals have been put forward in the literature. For later convenience we recall the well-known Bardina similarity model [9], given by

$$
m_{i j}^{B}=\left[L, \Pi_{i j}\right](\overline{\mathbf{u}})=\overline{\bar{u}}_{j} \bar{u}_{i}-\overline{\bar{u}}_{j} \overline{\bar{u}}_{i} .
$$

We observe that in this model the definition of the turbulent stress tensor in (10) is applied to the filtered solution, to obtain closure. A closely related modification of Bardina's model $[8,9]$ arises as

$$
m_{i j}^{m B}=\overline{\bar{u}}_{j} \bar{u}_{i}-\bar{u}_{j} \bar{u}_{i}
$$


which was proposed and studied in [22, 23] and recently further analyzed in [21]. Generalizations that include an approximate inversion of the spatial filter were put forward in $[34,37]$. A comprehensive discussion of models based on (approximate) deconvolution may be found in [23].

An alternative closure approach for the turbulent stress tensor is obtained by the so-called direct regularization of the convective flux. The models that will be considered in this paper can concisely be formulated, starting from the modified momentum equation

$$
\partial_{t} u_{i}+\partial_{j}\left(v_{j} w_{i}\right)+\partial_{i} p-\frac{1}{R e} \partial_{j j} u_{i}=0 .
$$

This equation governs the evolution of the velocity field $\mathbf{u}$ as a result of standard viscous fluxes and pressure gradient, but with an extended definition of the convective fluxes. We will refer to $\mathbf{v}$ as the 'convecting' velocity field and $\mathbf{w}$ as the 'convected' velocity field. As an example, if we adopt $v_{i}=w_{i}=u_{i}$ for $i=1,2,3$, we re-obtain the standard momentum equations. Different regularization models correspond to specific choices for $\mathbf{v}$ and $\mathbf{w}$. The simplest regularization models that we consider here are

$$
\begin{aligned}
& \text { Leray : } \quad v_{i}=\bar{u}_{i} ; w_{i}=u_{i}, \\
& \text { Modified Leray: } \quad v=u_{i} ; w_{i}=\bar{u}_{i}, \\
& \text { Modified Bardina: } \quad v_{i}=\bar{u}_{i} ; w_{i}=\bar{u}_{i} .
\end{aligned}
$$

The Leray regularization follows from the seminal paper of Leray [11], used in the context of large-eddy simulation in [12,14]. The modified Leray regularization was proposed in [24] and the simplified Bardina model was put forward in [21-23]. Each of these modifications of the convective fluxes induces a particular sub-filter model to be used in the large-eddy template. If we filter (13) then we obtain

$$
\partial_{t} \bar{u}_{i}+\partial_{j}\left(\bar{u}_{j} \bar{u}_{i}\right)+\partial_{i} \bar{p}-\frac{1}{R e} \partial_{j j} \bar{u}_{i}=-\partial_{j} m_{i j}^{R},
$$

where the regularization model tensors $m_{i j}^{R}$ are given by

$$
m_{i j}^{R}=\overline{v_{j} w_{i}}-\bar{u}_{j} \bar{u}_{i}
$$

In detail, we hence obtain

$$
\begin{aligned}
& \text { Leray : } m_{i j}^{L}=\overline{\bar{u}}_{j} u_{i}-\bar{u}_{j} \bar{u}_{i}, \\
& \text { Modified Leray: } \quad m_{i j}^{m L}=\overline{u_{j} \bar{u}_{i}}-\bar{u}_{j} \bar{u}_{i}, \\
& \text { Modified Bardina: } \quad m_{i j}^{m B}=\overline{\bar{u}}_{j} \bar{u}_{i}-\bar{u}_{j} \bar{u}_{i} .
\end{aligned}
$$

The regularization models listed above are appealing from a mathematical point of view. For each of these models, the rigorous existence, uniqueness and regularity of the solution to the modeled equations have been established [11, 21-24].

Whenever the unfiltered solution $\mathbf{u}$ appears in one of the regularization model tensors, we imply that an approximate inversion of the Helmholtz filter is used. Specifically, $\mathbf{u}$ is replaced by $\mathcal{L}(\overline{\mathbf{u}})$ where $\mathcal{L}$ denotes the approximate inverse of $L$. Various approximate inversion procedures have been suggested in the literature. A polynomial inversion of order $n$ is obtained by requiring $\mathcal{L}\left(L\left(x^{k}\right)\right)=x^{k}$ for $k=0, \ldots, n$ [37]. A related procedure is known as the approximate deconvolution method (ADM) [34]. These approximation procedures are applicable for general graded filters [38]. Here, we consider the Helmholtz filter, which 
can be inverted exactly at each computational resolution. Since the flow simulations adopt a (pseudo-)spectral formulation the inversion of the Helmholtz filter is straightforward. In fact, if we start from a Fourier series for $\bar{u}_{i}$ with coefficients $\left\{\bar{c}_{k}\right\}$ then the reconstructed solution that enters (19) or (20) has Fourier coefficients $\left\{\bar{c}_{k} / \mathcal{H}(k \Delta)\right\}$. Thus, multiplication with a modedependent factor $1 / \mathcal{H}(k \Delta)$ provides a simple implementation of the inverse of the Helmholtz filter.

An extended regularization principle, known as the NS- $\alpha$ model, may be obtained under certain conditions (see [17, 39]) by starting from the following Kelvin theorem:

$$
\frac{\mathrm{d}}{\mathrm{d} t}\left(\oint_{\Gamma(\mathbf{u})} u_{i} \mathrm{~d} x_{i}\right)-\frac{1}{R e} \oint_{\Gamma(\mathbf{u})} \partial_{j j} u_{i} \mathrm{~d} x_{i}=0,
$$

where $\Gamma(\mathbf{u})$ is a closed fluid loop moving with the Eulerian velocity $\mathbf{u}$. The unfiltered NavierStokes equations may be derived from $(22)$ [4, 17]. This provides some of the inspiration for an alternative regularization principle for the Navier-Stokes turbulence [17]. In fact, the so-called NS- $\alpha$ regularization principle was originally proposed on the basis of Taylor's hypothesis of frozen-in turbulence in a Lagrangian averaging framework [40, 41]. In the NS- $\alpha$ model, the fluid loop is considered to move with the smoothed transport velocity $\overline{\mathbf{u}}$, although the circulation velocity is still the unsmoothed velocity, $\mathbf{u}$. That is, in (22) we replace $\Gamma(\mathbf{u})$ by $\Gamma(\overline{\mathbf{u}})$; so the material loop $\Gamma$ moves with the filtered transport velocity. From this filtered Kelvin principle, we may obtain the Euler-Poincaré equations governing the smoothed solenoidal fluid dynamics [41], i.e., $\partial_{j} \bar{u}_{j}=0$ and

$$
\partial_{t} u_{i}+\partial_{j}\left(\bar{u}_{j} u_{i}\right)+u_{j} \partial_{i} \bar{u}_{j}+\partial_{i} p-\partial_{i}\left(\frac{1}{2} \bar{u}_{j} u_{j}\right)-\frac{1}{R e} \partial_{j j} u_{i}=0 .
$$

Comparison with the Leray regularization principle in (14) reveals two additional terms in (23). These terms guarantee the regularized flow to be consistent with the modified Kelvin circulation theorem in which $\Gamma(\mathbf{u}) \rightarrow \Gamma(\overline{\mathbf{u}})$.

The Euler-Poincaré equations (23) can also be rewritten in the form of the LES template. The implied sub-filter model is given by

$\partial_{t} \bar{u}_{i}+\partial_{j}\left(\bar{u}_{j} \bar{u}_{i}\right)+\partial_{i} \bar{p}-\frac{1}{R e} \partial_{j j} \bar{u}_{i}=-\partial_{j}\left(\overline{\bar{u}_{j} u_{i}}-\bar{u}_{j} \bar{u}_{i}\right)-\frac{1}{2}\left(\overline{u_{j} \partial_{i} \bar{u}_{j}-\bar{u}_{j} \partial_{i} u_{j}}\right)$.

We observe that the Leray model (19) reappears as a part of the NS- $\alpha$ sub-filter model on the right-hand side of (24). This formulation is given in terms of a general filter $L$ and its inverse. The sub-filter model presented in (24) can be specified further in case the filter $L$ has the Helmholtz operator as its inverse [17]. After some rewriting, the following parameterization for the turbulent stress tensor is obtained [28]:

$$
m_{i j}^{\mathrm{NS}-\alpha}=\alpha^{2} L\left(\partial_{k} \bar{u}_{i} \partial_{k} \bar{u}_{j}+\partial_{k} \bar{u}_{i} \partial_{j} \bar{u}_{k}-\partial_{i} \bar{u}_{k} \partial_{j} \bar{u}_{k}\right) .
$$

The first term on the right-hand side is the Helmholtz-filtered tensor-diffusivity model [42]. The second term combined with the first term, corresponds to the Leray regularization using the Helmholtz inversion as a filter. The third term completes the NS- $\alpha$ model and maintains Kelvin's circulation theorem. The global regularity of the NS- $\alpha$ model was established in [18]. In addition, an estimate for the dimension of the global attractor was obtained and related to the number of degrees of freedom in $[17,18]$.

In the following section, we describe the numerical method used to assess the regularization models. We also provide a general impression of the flow modifications that arise from the use of the different sub-filter models. The consequences of these modifications for statistical flow properties will be considered in section 4 . 


\section{The numerical simulation of decaying homogeneous isotropic turbulence}

We use a pseudo-spectral discretization combined with explicit time-stepping as described in section 3.1. Impressions of the large-eddy solutions obtained with the regularization models are collected in section 3.2.

\subsection{The pseudo-spectral discretization of regularized incompressible flow}

We will specify the pseudo-spectral discretization of the Navier-Stokes equations first and then describe the extensions required to treat the various regularization models introduced above. We use a numerical method in which no explicit Poisson system for the pressure needs to be solved. Closely following [3, 43] we express the Navier-Stokes equations in vector notation as

$$
\partial_{t} \mathbf{u}(\mathbf{x}, t)+\mathbf{u}(\mathbf{x}, t) \cdot \nabla \mathbf{u}(\mathbf{x}, t)=-\nabla p(\mathbf{x}, t)+\frac{1}{R e} \nabla^{2} \mathbf{u}(\mathbf{x}, t) .
$$

We may rewrite the nonlinear convective flux, thanks to the incompressibility condition, as

$$
\mathbf{u}(\mathbf{x}, t) \cdot \nabla \mathbf{u}(\mathbf{x}, t)=\boldsymbol{\omega}(\mathbf{x}, t) \times \mathbf{u}(\mathbf{x}, t)+\frac{1}{2} \nabla|\mathbf{u}(\mathbf{x}, t)|^{2},
$$

where $\boldsymbol{\omega}(\mathbf{x}, t)=\nabla \times \mathbf{u}(\mathbf{x}, t)$ is the vorticity. Substituting this into (26) and introducing the nonlinear term as $\mathbf{W}(\mathbf{x}, t)=\mathbf{u}(\mathbf{x}, t) \times \boldsymbol{\omega}(\mathbf{x}, t)$, we may write

$$
\left(\partial_{t}-\frac{1}{R e} \nabla^{2}\right) \mathbf{u}(\mathbf{x}, t)=\mathbf{W}(\mathbf{x}, t)-\nabla\left(p(\mathbf{x}, t)+\frac{1}{2}|\mathbf{u}(\mathbf{x}, t)|^{2}\right) .
$$

The flow is solved subject to periodic boundary conditions in all directions.

The system of equations (28) with incompressibility constraint $\nabla \cdot \mathbf{u}=0$ can be easily transformed to spectral space. Each of the velocity components $u_{\alpha}(\mathbf{x}, t)$ is expanded in terms of Fourier series dependent on the wavevector $\mathbf{k}$ :

$$
u_{\alpha}(\mathbf{x}, t)=\sum_{\mathbf{k}} u_{\alpha}(\mathbf{k}, t) \mathrm{e}^{\mathrm{i} \mathbf{k} \cdot \mathbf{x}} .
$$

Here, and in the sequel we use $\mathbf{k}$ to denote the wavevector. Note that this differs from its use in (4) where it denotes the vector of integer wavenumbers. We use the same notation for quantities in the physical and the spectral space, indicating the difference only by $\mathbf{x}$ or $\mathbf{k}$ as variables [3]. The transformed equation has the form

$$
\left(\partial_{t}+\frac{1}{R e} k^{2}\right) \mathbf{u}(\mathbf{k}, t)=\mathbf{W}(\mathbf{k}, t)-\mathrm{ik} \mathcal{F}\left(p(\mathbf{x}, t)+\frac{1}{2}|\mathbf{u}(\mathbf{x}, t)|^{2}, \mathbf{k}\right),
$$

where $\mathcal{F}(a(\mathbf{x}, t), \mathbf{k})$ denotes the Fourier coefficient of the function $a(\mathbf{x}, t)$ corresponding to the wavevector $\mathbf{k}$ :

$$
\mathcal{F}(a(\mathbf{x}, t), \mathbf{k})=A(\mathbf{k}, t) \quad \text { if } \quad a(\mathbf{x}, t)=\sum_{\mathbf{k}} A(\mathbf{k}, t) \mathrm{e}^{\mathrm{i} \mathbf{k} \cdot \mathbf{x}} .
$$

Likewise, $\mathbf{W}(\mathbf{k}, t)$ denotes the kth Fourier coefficient of the nonlinearity $\mathbf{W}(\mathbf{x}, t)$.

To eliminate the pressure term, and hence the need for a separate time-consuming Poisson equation solver, (30) is multiplied by $\mathbf{k}$, which corresponds to taking the divergence of (28) in physical space. Using the continuity equation in spectral space $(\mathbf{k} \cdot \mathbf{u}(\mathbf{k}, t)=0)$, the pressure term can be written as

$$
\mathcal{F}\left(p(\mathbf{x}, t)+\frac{1}{2}|\mathbf{u}(\mathbf{x}, t)|^{2}, \mathbf{k}\right)=\frac{\mathbf{k} \cdot \mathbf{W}(\mathbf{k}, t)}{\mathrm{i} k^{2}} .
$$


This leads to the final form of the system of equations:

$$
\left(\frac{\partial}{\partial t}+\frac{1}{R e} k^{2}\right) \mathbf{u}(\mathbf{k}, t)=\mathbf{W}(\mathbf{k}, t)-\frac{\mathbf{k}}{k^{2}} \mathbf{k} \cdot \mathbf{W}(\mathbf{k}, t) \equiv \mathbf{D W}(\mathbf{k}, t),
$$

where in the last step we introduced the projection operator $\mathbf{D}: D_{\alpha \beta}(\mathbf{k})=\delta_{\alpha \beta}-k_{\alpha} k_{\beta} / k^{2}$, projecting $\mathbf{W}$ onto the plane normal to the wavevector $\mathbf{k}$. For a detailed discussion of this approach see [3, 44].

We incorporate various models in the nonlinear term substituting $\mathbf{W}(\mathbf{k}, t)$ by its modified version $\overline{\mathbf{W}}(\mathbf{k}, t)$ :

$$
\begin{aligned}
& \text { Leray: } \quad \overline{\mathbf{W}}(\mathbf{k}, t)=-\mathcal{F}((\overline{\mathbf{u}}(\mathbf{x}, t) \cdot \nabla) \mathbf{u}(\mathbf{x}, t), \mathbf{k}), \\
& \text { Modified Leray: } \quad \overline{\mathbf{W}}(\mathbf{k}, t)=-\mathcal{F}((\mathbf{u}(\mathbf{x}, t) \cdot \nabla) \overline{\mathbf{u}}(\mathbf{x}, t), \mathbf{k}) \text {, } \\
& \text { Modified Bardina: } \quad \overline{\mathbf{W}}(\mathbf{k}, t)=-\mathcal{F}((\overline{\mathbf{u}}(\mathbf{x}, t) \cdot \nabla) \overline{\mathbf{u}}(\mathbf{x}, t), \mathbf{k}) \text {, } \\
& \text { NS- } \alpha: \quad \overline{\mathbf{W}}(\mathbf{k}, t)=-\mathcal{F}((\overline{\mathbf{u}}(\mathbf{x}, t) \cdot \nabla) \mathbf{u}(\mathbf{x}, t), \mathbf{k}) \\
& -\mathcal{F}\left(\sum_{j=1}^{3} u_{j}(\mathbf{x}, t) \nabla \bar{u}_{j}(\mathbf{x}, t), \mathbf{k}\right) \text {. }
\end{aligned}
$$

Hence, the modification of the Navier-Stokes equations needed to accommodate the different regularization models involves the convective nonlinearity only. This is readily implemented in the pseudo-spectral framework.

To solve (33) we use the fact that the diffusive terms can be computed exactly by introducing an integrating factor $\mathrm{e}^{k^{2} t / R e}$. In fact, we may concisely write

$$
\partial_{t} \mathbf{U}(\mathbf{k}, t)=\mathbf{f}(\mathbf{U}(\mathbf{k}, t)),
$$

where

$$
\mathbf{U}=\left\{u_{\alpha}(\mathbf{k}, t) \mathrm{e}^{k^{2} t / R e}\right\}, \quad \mathbf{f}=\left\{D_{\alpha \beta} W_{\beta}(\mathbf{k}, t) \mathrm{e}^{k^{2} t / R e}\right\} .
$$

Introducing the time-step $\Delta t$, so that $t^{n}=n \Delta t$, we can integrate this problem using the four-stage compact storage Runge-Kutta method:

$$
\mathbf{U}\left(\mathbf{k}, t^{n+\gamma}\right)=\mathbf{U}\left(\mathbf{k}, t^{n}\right)+\gamma \Delta t \mathbf{f}\left(\mathbf{U}\left(\mathbf{k}, t^{n+\xi}\right)\right)
$$

with coefficients $(\gamma, \xi) \in\{(1 / 4,0),(1 / 3,1 / 4),(1 / 2,1 / 3),(1,1 / 2)\}$ in the four consecutive stages of a time-step. The explicit final form of the discretized equations is

$u_{\alpha}\left(\mathbf{k}, t^{n+\gamma}\right)=u_{\alpha}\left(\mathbf{k}, t^{n}\right) \mathrm{e}^{-k^{2} \gamma \Delta t / R e}+\gamma \Delta t D_{\alpha \beta} W_{\beta}\left(\mathbf{k}, t^{n+\xi}\right) \mathrm{e}^{-k^{2}(\gamma-\xi) \Delta t / R e}$.

The method of numerical solution is pseudo-spectral, i.e., the spatial-derivative terms in the Navier-Stokes equations are computed in spectral space, while the nonlinear terms of the momentum equations are evaluated by back-transforming the velocities to the physical space to perform the required products of velocities. This also applies to the nonlinear terms present in the regularization models. There are different ways to eradicate the aliasing errors [45]. We applied the method with two shifted grids and spherical truncation [44, 46]. With this method the aliasing error is fully removed from the simulations, which is particularly relevant in case large-eddy simulations at comparably coarse resolutions are considered. The de-aliasing is done at the expense of doubling the costs of computations and memory usage. An efficient parallel implementation was developed to simulate the turbulent flow. A detailed validation of this code was reported in $[46,48]$.

\subsection{Flow structures in regularized turbulence}

In order to obtain a global impression of the predictions obtained with the regularization subfilter models, we present flow solutions in terms of typical snapshots of the velocity field. This 
allows us to roughly ascertain whether or not the instantaneous solutions 'resemble' the actual filtered direct numerical simulation findings as far as turbulent flow structures are concerned. This assessment is only quite crude and will be complemented with a quantitative analysis in the following section.

We consider turbulence in a cubic box of side $\ell$ with periodic boundary conditions. We use $N$ Fourier modes per coordinate direction, consistent with the assumed statistical isotropy. The wavevector $\mathbf{k}$ is given by: $k_{\alpha}=2 \pi n_{\alpha} / \ell$ for integer $n_{\alpha}=0, \pm 1, \pm 2, \ldots$, $\pm(N / 2-1),-N / 2$. The cut-off wavenumber is given by $k_{\max }=\pi N / \ell$. The initial condition is taken identical to [49], adopting an initial Taylor Reynolds number of $R e_{\lambda}=100$ and a computational Reynolds number $R e \approx 4000$ based on the domain-size.

We consider the flow that is predicted by the various models at a resolution of $N^{3}$ Fourier modes, with $N=128$. This corresponds to an approximately grid-independent solution [46], in particular when the filter-parameter $\alpha \geqslant \ell / 160$, i.e., at filter widths $\Delta \geqslant \ell / 32$. The ratio between the filter width $\Delta$ and the grid-spacing $h$ is known as the sub-filter resolution, which takes values $\Delta / h \geqslant 2-4$ in order to obtain near grid-independence, a value that was also observed in [50]. An impression of the velocity field is shown in figure 1. In the first row we observe the degree of flow coarsening arising from the application of the Helmholtz filter to the DNS data. This provides the point of reference with which the large-eddy simulations will be compared. Turning to the regularization models we observe from the first column of figures at $\alpha=\ell / 320$, i.e., $\Delta \approx \ell / 64$, that the sub-filter models do not contribute much to the total flux and the results can hardly be distinguished from the filtered DNS data. Turning to the second column at $\alpha=\ell / 160$, we note that the Leray prediction is somewhat coarsened, corresponding to the wider filter width. This is also observed in the NS- $\alpha$ model, albeit with a slight tendency to over-predict the small-scale variability of the solution, compared to the filtered DNS. This tendency is even stronger in the modified Leray model in which we may already appreciate from this simple inspection that the solution develops too many small scales. For $\alpha=\ell / 80$ these trends are expressed more strongly-we observe that the Leray prediction resembles the filtered DNS results quite closely, NS- $\alpha$ further over-represents small-scale variability and the modified Leray model is seen to yield very poor correspondence with the filtered DNS at this filter width. This may also be noticed from the last column of figures in which we observe, in particular, a complete disappearance of correlation between the modified Leray model and the filtered DNS. At this large filter width, both the Leray and NS- $\alpha$ models are still capturing the large-scale impression of the filtered solution. A similar general trend was observed in relation to the prediction of vorticity, although any (slight) over-prediction of small scales in the velocity has a much more pronounced influence on vorticity.

In the following section, we analyze some statistical properties of homogeneous turbulence in more detail, to establish the accuracy with which regularization models represent physical properties of the flow. The overestimation of the smaller scales, compared to the filtered DNS results, will prove to be an important limitation on the quality of predictions of the models, particularly at larger filter widths.

\section{Statistical properties of regularized homogeneous turbulence}

In this section we consider the prediction of three key statistical properties of homogeneous decaying isotropic turbulence as obtained with the different regularization models. First, we recall the main elements of energy dynamics in turbulent flow in section 4.1. Then we present the simulation results in section 4.2. We include a discussion of the reference direct numerical simulation and quantify the effect of Helmholtz filtering. Subsequently, we turn to the accuracy 

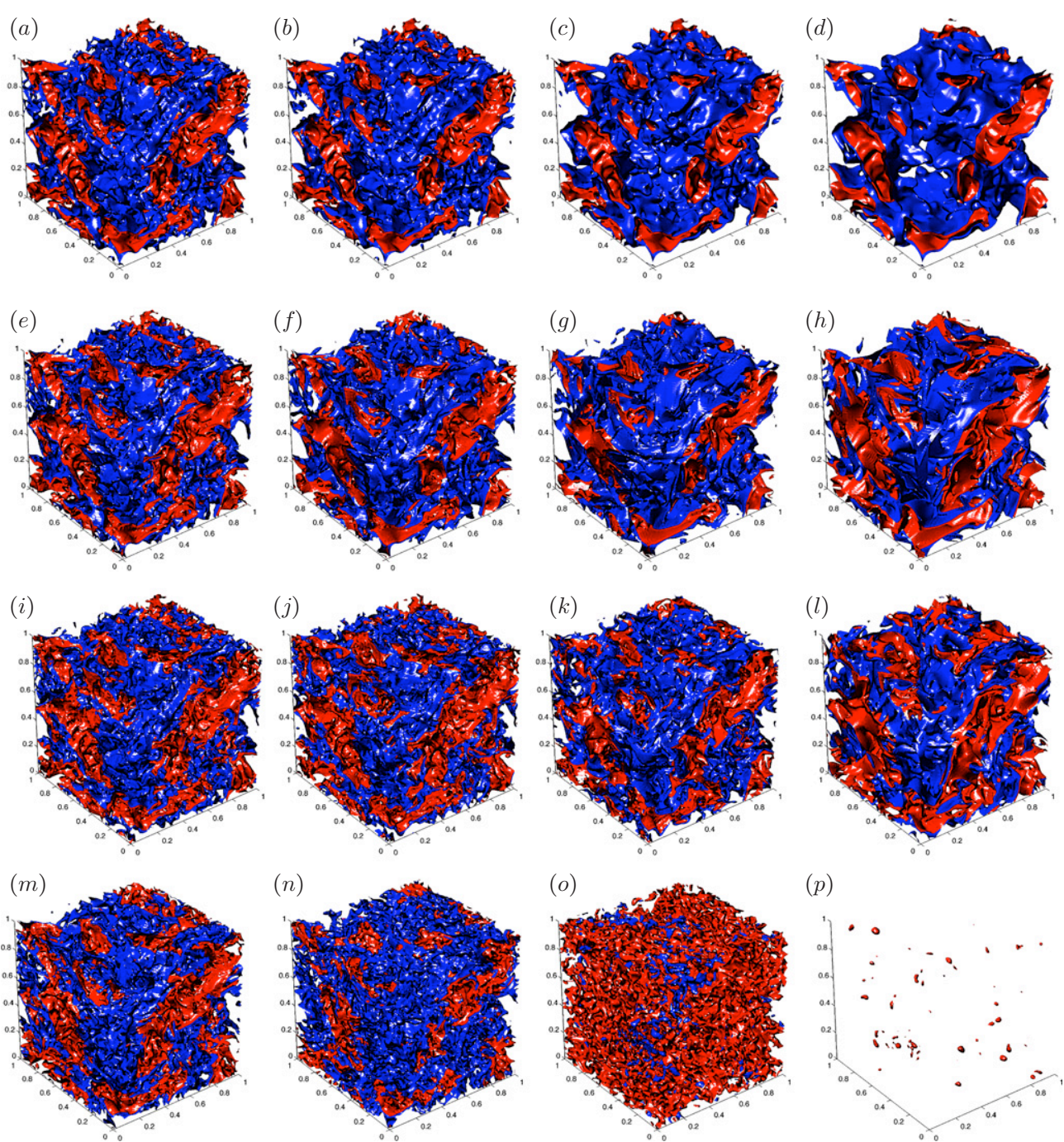

$(p)$

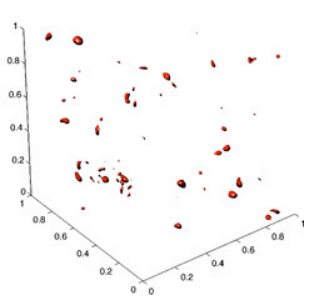

Figure 1. Instantaneous velocity field at $t=0.1$, showing the velocity component in the $x_{1}$ direction. Regions with a positive $u_{1}$ are colored red while the negative $u_{1}$ is shown in blue. We show results at $N^{3}$ grid points with $N=512$ for the direct numerical simulation and $N=128$ for the large-eddy simulations. We adopt the Helmholtz filter. Results from the filtered DNS ( $a, b, c$, $d)$, Leray $(e, f, g, h), \mathrm{NS}-\alpha(i, j, k, l)$ and the modified Leray model $(m, n, o, p)$ at $\alpha=\ell / 320(a, e$, $i, m), \alpha=\ell / 160(b, f, j, n), \alpha=\ell / 80(c, g, k, o)$ and $\alpha=\ell / 40(d, h, l, p)$ are shown.

with which the regularization models predict the primary features of homogeneous, isotropic turbulence at various filter widths and spatial resolutions.

\subsection{Elements of energy dynamics}

The energy dynamics in a turbulent flow is governed by the dissipation and transfer of energy. Changing to index notation we may rewrite (33) as

$$
\left(\partial_{t}+\frac{1}{R e} k^{2}\right) u_{\alpha}(\mathbf{k}, t)=D_{\alpha \beta}(\mathbf{k}) W_{\beta}(\mathbf{k}, t) \equiv \Psi_{\alpha}(\mathbf{k}, t) .
$$


Multiplying this set of equations by the complex-conjugate of the Fourier velocity component $u_{\alpha}^{*}(\mathbf{k}, t)$ and summing, we obtain the energy equation:

$$
\left(\partial_{t}+2 \frac{1}{R e} k^{2}\right) E(\mathbf{k}, t)=u_{\alpha}^{*}(\mathbf{k}, t) \Psi_{\alpha}(\mathbf{k}, t),
$$

where $E(\mathbf{k}, t)=\frac{1}{2}|\mathbf{u}(\mathbf{k}, t)|^{2}$ defines the kinetic energy distribution in spectral space. Introducing the notation for the rate of energy transfer $T(\mathbf{k}, t)=u_{\alpha}^{*}(\mathbf{k}, t) \Psi_{\alpha}(\mathbf{k}, t)$ and the dissipation rate $\varepsilon_{d}(\mathbf{k}, t)=2 k^{2} E(\mathbf{k}, t) / R e$, we can simplify this as

$$
\partial_{t} E(\mathbf{k}, t)=-\varepsilon_{d}(\mathbf{k}, t)+T(\mathbf{k}, t) .
$$

Since we deal with homogeneous, isotropic turbulence, the shell-averaged energy distribution $E(k, t)$ and transfer rate $T(k, t)$ will be considered. In addition, the total kinetic energy $\mathcal{E}$ and total dissipation rate $\varepsilon(t)$ will be studied. These are obtained by integrating $E(\mathbf{k}, t)$ and $\varepsilon_{d}(\mathbf{k}, t)$ over $\mathbf{k}$ [46]. In addition, we will consider the power spectrum $\Pi$, which is obtained as

$$
\Pi(k, t)=-\int_{0}^{k} T(\kappa, t) \mathrm{d} \kappa
$$

If a regularization model is analyzed we focus on the dominant contribution to $T(k, t)$ only, i.e., the contributions $T(\mathbf{k}, t)=\bar{u}_{\alpha}^{*}(\mathbf{k}, t) \Psi_{\alpha}(\mathbf{k}, t)$. Together, these quantities characterize in some detail the large- and small-scale contributions to the dynamics of the kinetic energy. These provide a good basis for the assessment of the regularization models.

In this paper, we compare the energy dynamics of the regularization models to that associated with the filtered DNS solution. We consider the dynamics of the resolved energy, i.e., the energy based on the filtered representation $\left|\bar{u}_{\alpha}(\mathbf{k}, t)\right|$. This method of comparison is directly motivated by the large-eddy simulation framework [4, 5]. Alternatively, one could consider the explicit energy balances associated with the regularization models. This involves slight re-definitions of the quantity referred to as the kinetic energy, and differs from model to model. At small values of the filter width, the differences can be neglected, but for wider filters these re-interpretations of the 'kinetic energy' do show some differences with the resolved kinetic energy. This alternative comparison was studied in [47].

In the assessment of the regularization models we also include the evolution of two flow properties that are closely related to the energy dynamics. First, the Taylor Reynolds number $R e_{\lambda}$ defined as

$$
R e_{\lambda}(t)=\operatorname{Re} u_{\mathrm{rms}}(t) \lambda(t)
$$

is considered. Here,

$$
u_{\mathrm{rms}}(t)=\sqrt{2 \mathcal{E}(t) / 3}
$$

denotes the root-mean-square velocity fluctuation and $\lambda$ is the Taylor microscale given by

$$
\lambda(t)=\left[5 \mathcal{E}(t) / \int_{0}^{k_{\max }} k^{2} E(k, t) \mathrm{d} k\right]^{1 / 2}=\frac{\sqrt{\left\langle u^{2}(\mathbf{x}, t)\right\rangle}}{\sqrt{\left\langle\left(\partial_{1} u(\mathbf{x}, t)\right)^{2}\right\rangle}}
$$

in which $k_{\max }$ is the largest wavenumber in the computation and $\langle\cdot\rangle$ denotes volume averaging over the entire flow domain. Moreover, to scrutinize the quality with which the smaller scales are represented, the skewness is included:

$$
S(t)=\frac{2}{35}\left(\frac{\lambda(t)}{u_{\mathrm{rms}}(t)}\right)^{3} \int_{0}^{k_{\max }} k^{2} T(k, t) \mathrm{d} k=\frac{\left\langle\left(\partial_{1} u(\mathbf{x}, t)\right)^{3}\right\rangle}{\left\langle\left(\partial_{1} u(\mathbf{x}, t)\right)^{2}\right\rangle^{3 / 2}}
$$


in terms of the shell-averaged energy-transfer spectrum $T(k, t)$. The flow properties $\mathcal{E}, \operatorname{Re}_{\lambda}$ and $S$ will next be used to illustrate the accuracy with which different parts of the dynamic range of the turbulent flow are predicted. The resolved kinetic energy is strongly influenced by the larger scales in the flow, whereas $R e_{\lambda}$ and $S$ depend on the components of the velocity derivatives and hence characterize the accuracy with which smaller scales are captured.

\subsection{Comparison of regularization LES with filtered DNS}

In this subsection, we first consider the reference direct numerical simulation and the effect of Helmholtz filtering. Then we turn to a detailed comparison of predictions based on regularization models with filtered DNS.

4.2.1. Grid-independence of direct numerical simulation. In the following, we will validate predictions of the regularization models against data obtained from direct numerical simulations. Therefore, it is of importance to establish the quality of the DNS through a gridconvergence study. This also gives basic information on the effect of coarse spatial resolution in the large-eddy simulations that will be presented momentarily. In figure 2, we show the convergence of $\left(\mathcal{E}, \operatorname{Re}_{\lambda}, S\right)$. For each of these three flow properties a clear convergence is observed, approximating a truly grid-independent solution. We observe significant deviations from the grid-independent solution at coarse resolutions $N=32$ and 64. Conversely, $\mathcal{E}$ and $R e_{\lambda}$ are very well approximated at $N=128$, while the velocity skewness $S$ requires at least $N=256$ to be captured with high accuracy.

4.2.2. The effect of Helmholtz filtering. To assess the quality of the large-eddy predictions, it is required to compare results against filtered direct numerical simulations. We compare the effect of filtering at a range of Helmholtz-lengths, i.e., $\alpha$ in (4) varies from $\ell / 320$ to $\ell / 10$. This corresponds to effective filter widths $\Delta=\ell / 64, \ldots, \ell / 2$. The strongest filtering at $\Delta \approx \ell / 2$ was deliberately chosen well inside the largest flow scales to also assess the limitations of regularization models in the case of so-called VLES (very-large-eddy simulations [1]).

In figure 3 we compiled the filtered DNS data. The procedure to obtain these results is as follows: (i) filter the DNS solution obtained at $N=512$ with a Helmholtz filter using the parameter $\alpha$, (ii) inject the filtered solution onto a grid at $N=128$ and (iii) on this grid, evaluate the corresponding flow property. The choice for evaluating the filtered solution at $N=128$ was made since at this resolution all the so-called sub-filter resolutions $r=\Delta / h \geqslant 2$. Values of $r \geqslant 2$ correspond (quite) closely to grid-independent LES [50], which serves as an important point of reference for assessing the quality of the regularization models without any contamination from numerics that could arise at coarse resolutions [49, 51, 52].

We clearly observe in figure 3 the strong influence of filtering on the solution. An increase in the filter width implies a monotonous reduction of the decay of the turbulent kinetic energy and the skewness, consistent with the stronger attenuation of more of the smaller scales. At sufficiently small filter widths, this monotonous trend also applies to the Taylor Reynolds number. In that case, we observe $R e_{\lambda}$ to increase with an increasing filter width, which is due to the fact that the increase in the Taylor micro-scale is stronger with an increasing filter width than the decrease of the root-mean-square fluctuations of the velocity $u_{\text {rms }}$. However, at very large filter widths we note the reverse trend. Once the filter width becomes of the size of the largest flow structures, the kinetic energy, and hence $u_{\text {rms }}$ (cf (44)), decrease more rapidly than can be compensated by an increase in $\lambda$. In total, this implies lower Taylor Reynolds numbers at very large filter widths. 
(a)

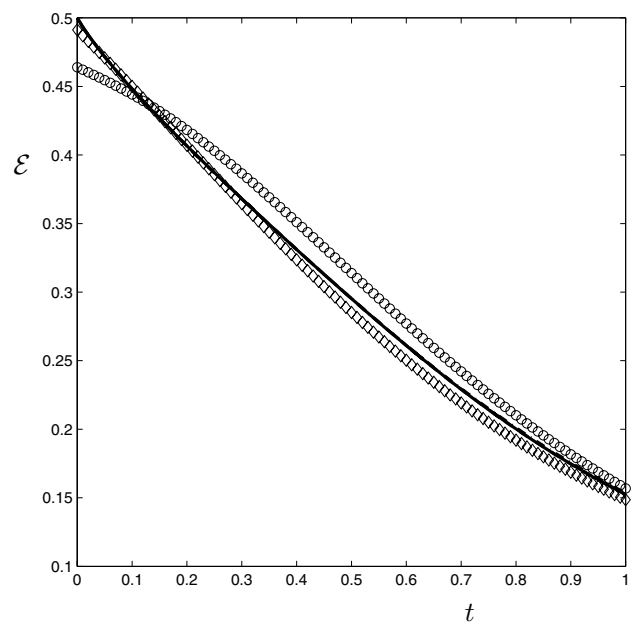

(b)

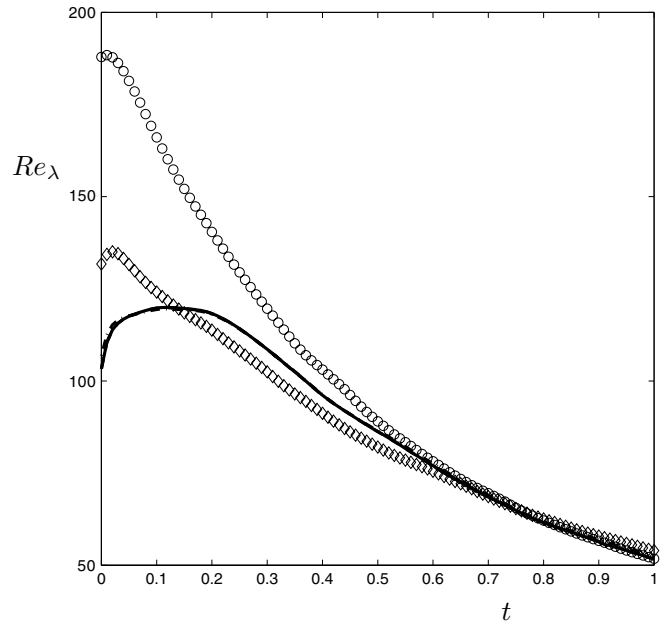

(c)

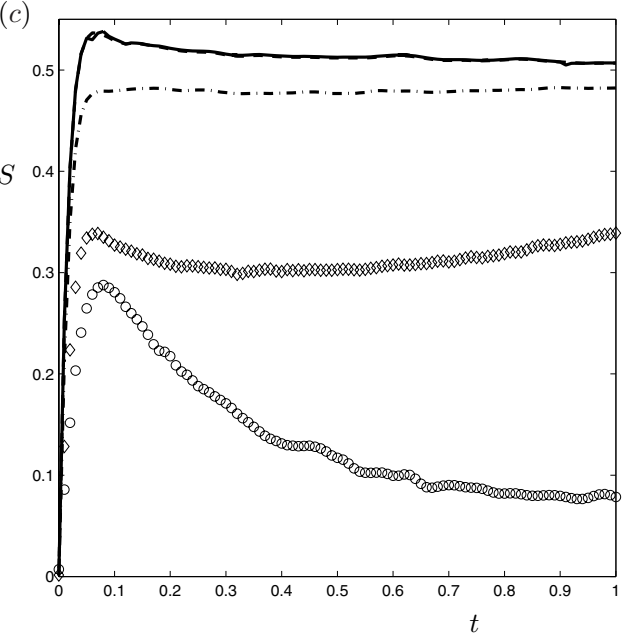

Figure 2. Evolution of the total kinetic energy $\mathcal{E},(a)$ the Taylor Reynolds number $R e_{\lambda}$ and (b) the skewness $S$, (c) quantifying the convergence toward DNS at resolutions $N^{3}$ with $N=$ $32(\circ), N=64(\diamond), N=128$ (dash-dot), $N=256$ (dash) and $N=512$ (solid).

We proceed with the actual assessment of the regularization models. The different simulations were initialized as follows. The initial field used for DNS at $N=512$ was filtered with the Helmholtz filter at the $\alpha$-value selected for the LES, and subsequently injected onto the chosen LES grid. This assures that at $t=0$ the flow properties evaluated from any of the large-eddy simulations and the filtered, injected DNS are identical. Various values of $\alpha$ and various spatial resolutions were included in this study. We distinguish between results obtained at sufficiently high sub-filter resolution $\Delta / h$, from coarsely resolved simulations. Grid-independent solutions allow us to assess how well the proposed model captures the primary flow features. This requires high resolution, for which we adopt simulations at $N=128$, thereby guaranteeing the $\Delta / h \geqslant 2$ for all simulations considered. Next to this 'academic LES testing' of the sub-filter modeling quality, we consider the influence of spatial resolution at coarse grids on the obtained results. 
$(a)$

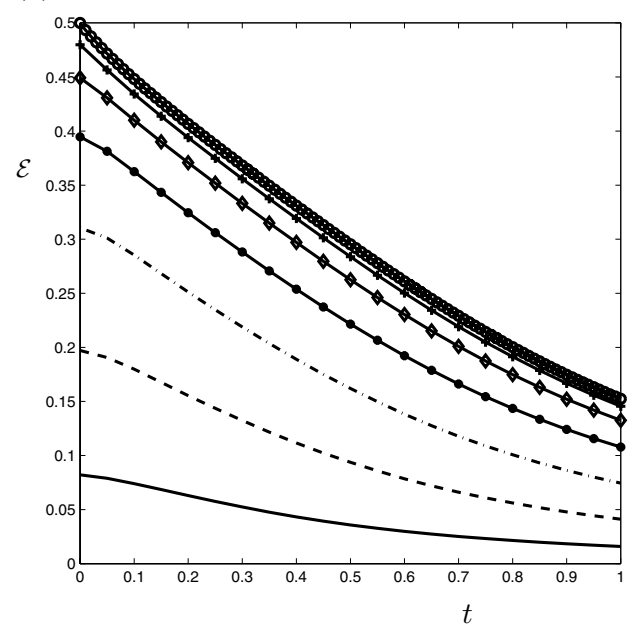

(b)

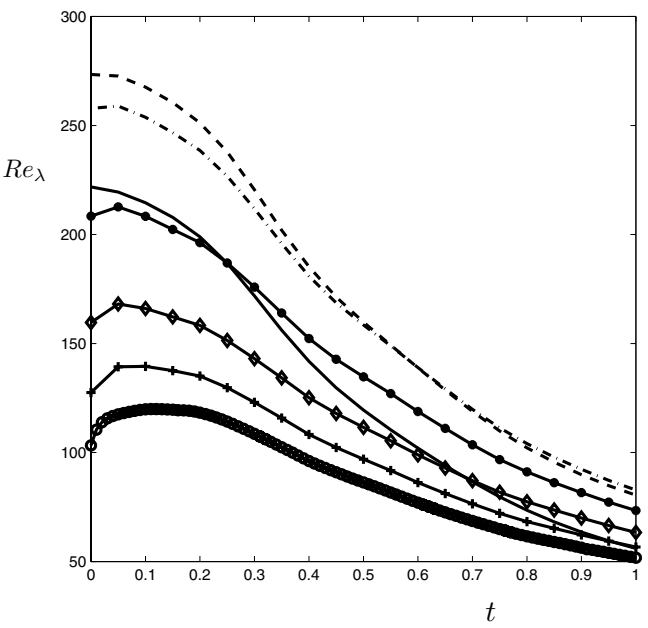

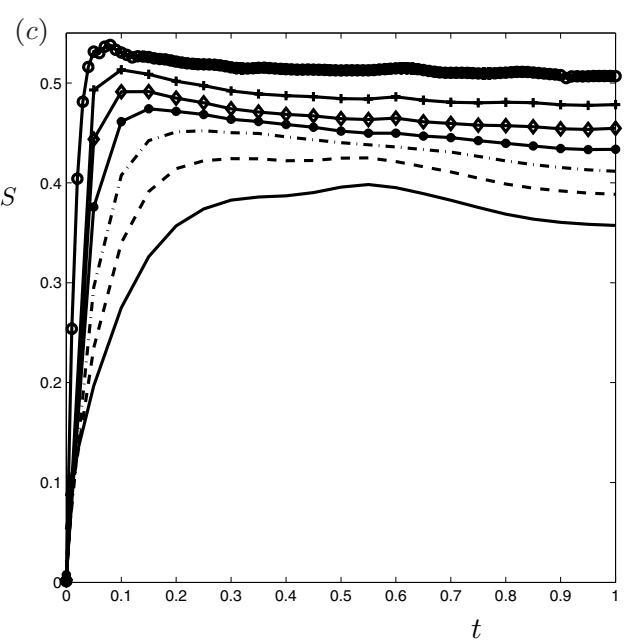

Figure 3. Evolution of the total kinetic energy $\mathcal{E}$, (a) the Taylor Reynolds number $R e_{\lambda}$ and $(b)$ the skewness $S,(c)$ quantifying the effect of Helmholtz filtering the DNS data obtained at resolution $N^{3}$ with $N=512$. The filtered DNS data were obtained with $\alpha=\ell / 10$ (solid), $\alpha=\ell / 20$ (dash), $\alpha=\ell / 40$ (dash-dot), $\alpha=\ell / 80$ (*), $\alpha=\ell / 160(\diamond), \alpha=\ell / 320$ (+) and $\alpha=0$ (०), and subsequently injected on a grid of $N^{3}$ with $N=128$ after which the quantity was evaluated.

4.2.3. Grid-independent LES predictions. In figure 4 we collected the results of the gridindependent LES predictions of the decay of the resolved kinetic energy, for all regularization models introduced. These results can be directly compared to the filtered DNS data, which are shown by the solid lines. For each value of $\alpha$ we note that the resolved kinetic energy is overpredicted - the contribution of the sub-filter model to the decay of energy is insufficient. This under-pins the observed small-scale variability in the solution seen in figure 1 and is reminiscent of the Bardina sub-filter model [9, 31].

The results of the various models correspond quite closely to each other at very small and at very large filter widths, albeit with very different implications. For small values of $\alpha$, the large-eddy predictions are close to the filtered DNS, primarily indicating that the sub-filter 
(a)

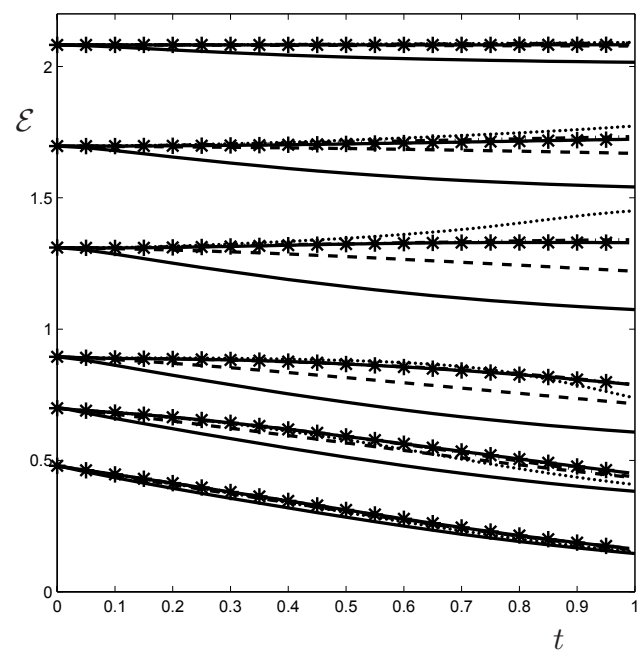

(b)

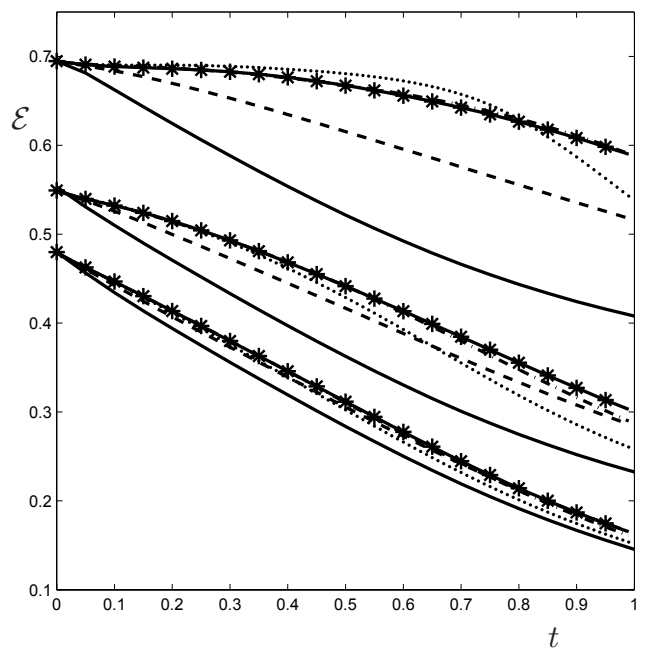

Figure 4. Decay of resolved kinetic energy obtained at $N=128$. In $(a)$ we show all cases considered, in $(b)$ we zoom in on the three cases with the smallest filter widths. Curves are labeled as follows: filtered DNS (solid), Leray (dash), NS- $\alpha$ (dash-dot), modified Leray (dot) and simplified Bardina (solid with *). Groups of curves correspond to $\alpha=\ell / 320, \ell / 160, \ell / 80, \ell / 40, \ell / 20$ and $\alpha=\ell / 10$ from bottom to top. To distinguish the groups of curves the latter five were shifted (from bottom to top) by $0.25,0.5,1,1.5$ and 2 , respectively, in figure (a) and the latter two by 0.1 and 0.3 , respectively, in figure $(b)$.

models do not contribute much to the dynamics of the simulated flow. As the filter width increases, we note that the different sub-filter models give quite different results. These follow the main trend in the energy decay as long as $\Delta \lesssim \ell / 32$. The underprediction of the energy decay-rate becomes more striking with increasing $\alpha$; the modified Leray model can even lead to a considerable increase of $\mathcal{E}$ for certain values of the filter width. The simplified Bardina and the NS- $\alpha$ models give almost identical results in which the decay of $\mathcal{E}$ is seen to be very small. The Leray model appears to represent at least some part of the desired energy decay, even at quite large filter widths. At very large filter widths, all models are seen to lead to simulations in which the resolved kinetic energy is almost constant. In this VLES regime, the regularization models do not capture the energy decay-rate.

In figure 5 the corresponding predictions of the decay of the Taylor Reynolds number and the skewness are collected, showing the dependence on the filter width for the individual sub-filter models. Generally speaking, we observe similar trends as in figure 4, i.e., the predictions become progressively less accurate with an increasing filter width. In the VLES regime, all models are found to yield large errors. However, in the 'LES regime' where $\Delta \lesssim O(\ell / 16)-O(\ell / 8)$ the primary flow features are captured properly with the regularization models. Turning to the Taylor Reynolds number, we note that the filtered DNS results are well approximated in case $\Delta \lesssim \ell / 16$. The predictions are found to be most accurate when the Leray model is used, while the errors are most pronounced for the modified Leray model. At large filter widths, particularly the simplified Bardina model displays strong deviations from the filtered DNS results. The skewness is seen to be predicted accurately by the Leray model for $\Delta \lesssim \ell / 8$. Here as well, we note that the modified Leray model yields rather large errors, while the NS- $\alpha$ and simplified Bardina models give quite comparable results. 
(a)

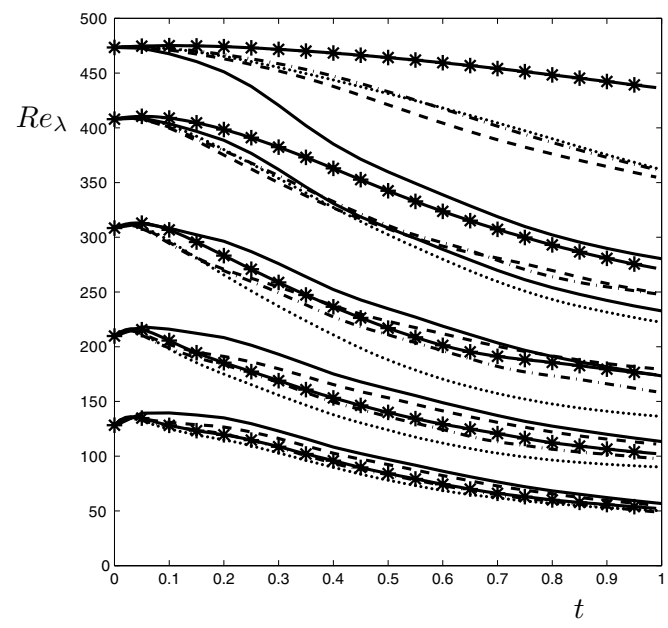

(b)

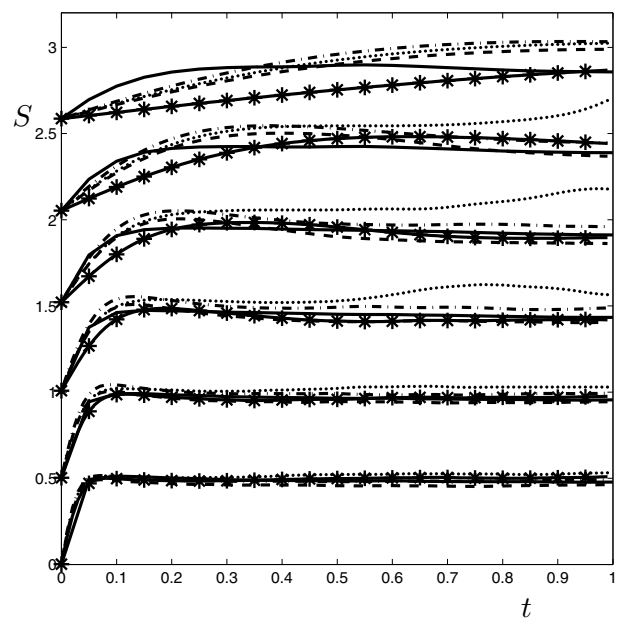

Figure 5. Decay of the Taylor Reynolds number $R e_{\lambda}(a)$ and the skewness $S(b)$ obtained at $N=128$. Curves are labeled as follows: filtered DNS (solid), Leray (dash), NS- $\alpha$ (dashdot), modified Leray (dot) and modified Bardina (solid with *). Groups of curves correspond to $\alpha=\ell / 320, \ell / 160, \ell / 80, \ell / 40$ and $\ell / 20$ from bottom to top in $(a)$ and also including $\ell / 10$ in $(b)$. To distinguish the groups of curves the latter four were shifted (from bottom to top) by 50, 100, 150 and 200, respectively, in figure $(a)$ and the latter five by $0.5,1,1.5,2$ and 2.5 , respectively, in figure $(b)$.

4.2.4. Simulation errors at coarse resolutions. In LES-practice one quite often does not have a proper grid-independent solution to the closed equations available $[4,54,55]$. It is therefore of importance to investigate to what extent the numerical solution is altered when the grid is coarsened. We illustrate this first for the Leray model at a variety of filter widths and then consider all other sub-filter models at a particular filter width.

In figure 6, we display the influence of under-resolution on the decay of the kinetic energy and the skewness. As may be expected, the effect of increasing the resolution is largest in cases with a small filter width. For the kinetic energy we note that a nearly gridindependent solution is obtained already at $N=32$ if $\alpha=\ell / 80$, i.e., $\Delta \approx \ell / 16$. At smaller filter widths $N=64$ is seen to be required, consistent with maintaining a suitable sub-filter resolution [50].

The effect of spatial discretization errors is much stronger for the skewness, as this quantity depends much more on small scales in the numerical solution. We note that the skewness is not predicted accurately for any of the 'LES-range' filter widths $\Delta \lesssim \ell / 8$ if a grid with $N=32$ is adopted. The skewness clearly requires higher sub-filter resolutions to achieve gridindependence. We note that the effect of spatial discretization errors is the dominant limitation for the Leray model at coarse resolution. In practice, a balance needs to be found between modeling errors, which become larger with an increasing filter width, and discretization errors, which become smaller with increasing filter widths [56, 57]. A reasonable choice in this case appears $N=64$ at $\Delta \approx O(\ell / 16)-O(\ell / 8)$. We infer a partial cancellation of modeling and discretization errors for the skewness. For low values of $\alpha$ an increase in $\alpha$ is seen to lead to a reduction of the integrated error, presumably since the discretization error is reduced, while at large $\alpha$ a further increase in $\alpha$ implies an increase in this error, presumably because of a continuing growth of the modeling error. This partial error-cancellation was also observed in $[49,57]$. 
(a)

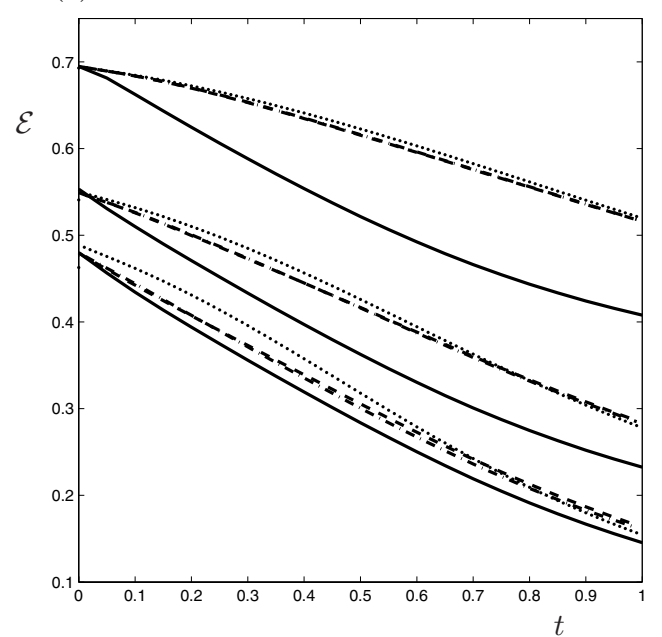

(b)

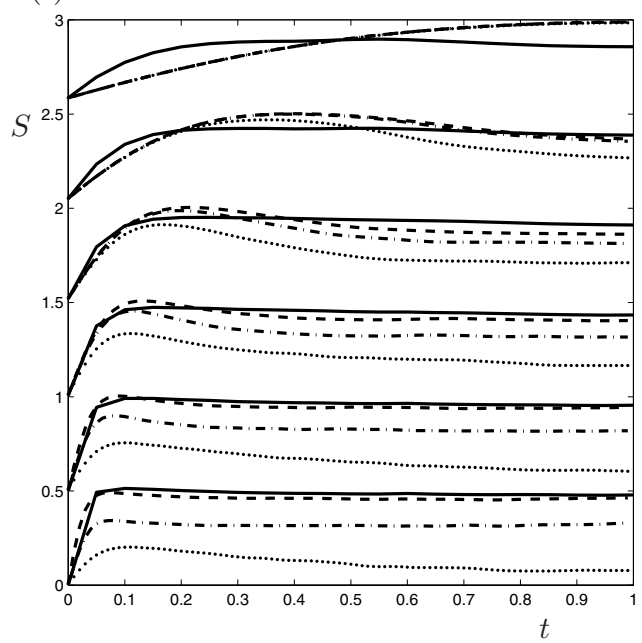

Figure 6. Decay of the resolved kinetic energy $\mathcal{E}(a)$ and the skewness $S(b)$ using the Leray model at spatial resolutions $N=128$ (dash), $N=64$ (dash-dot) and $N=32$ (dot). Filtered DNS data are shown as solid. Groups of curves correspond (from bottom to top) to $\alpha=\ell / 320, \ell / 160$ and $\ell / 80$ in $(a)$ and to $\alpha=\ell / 320, \ell / 160, \ell / 80, \ell / 40, \ell / 20$ and $\ell / 10$ in $(b)$. To distinguish the groups of curves the latter two were shifted (from bottom to top) by 0.1 and 0.3 , respectively, in figure $(a$ ) and the latter five by $0.5,1,1.5,2$ and 2.5 , respectively, in $(b)$.

(a)

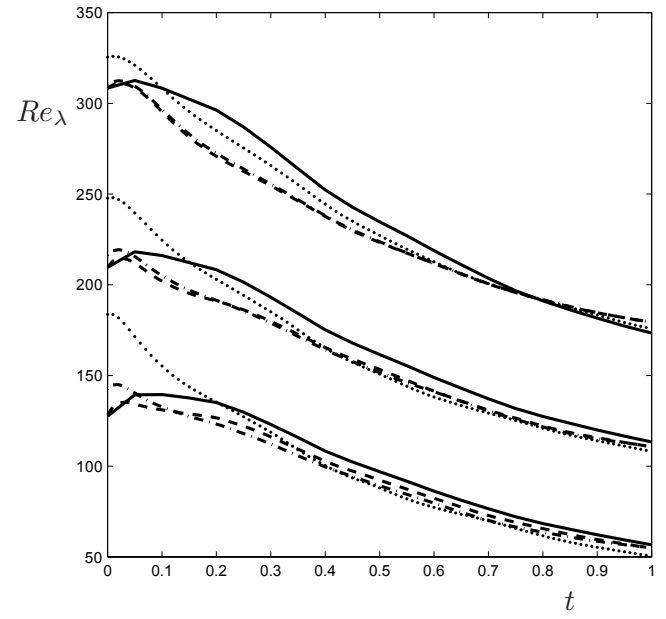

(b)

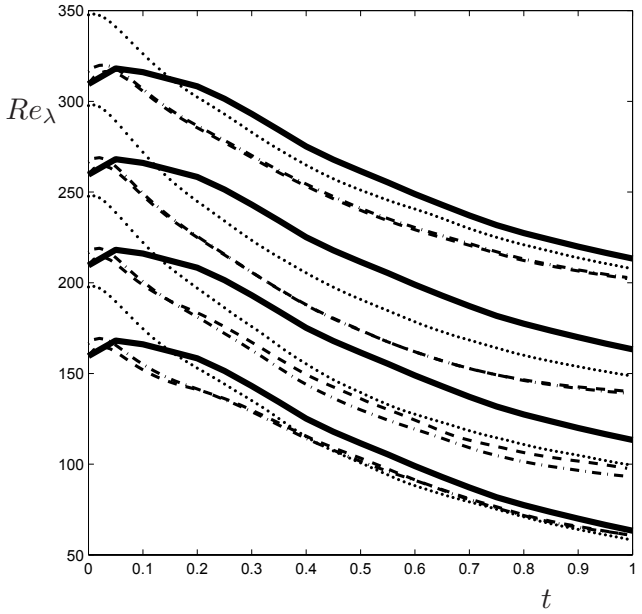

Figure 7. Decay of the Taylor Reynolds number $R e_{\lambda}$ using the Leray model in $(a)$ and the Leray, NS- $\alpha$, modified Leray and simplified Bardina models in $(b)$, at spatial resolutions $N=128$ (dash), $N=64$ (dash-dot) and $N=32$ (dot). Filtered DNS data are shown as solid. Groups of curves correspond to $\alpha=\ell / 320, \ell / 160$ and $\ell / 80$ from bottom to top in $(a)$. To distinguish the groups of curves the latter two were shifted by 50 and 100, respectively, in $(a)$. In $(b)$ we adopt $\alpha=\ell / 160$ and groups of curves correspond to Leray, NS- $\alpha$, modified Leray and simplified Bardina from bottom to top; the latter three were shifted by 50,100 and 150 , respectively.

The convergence toward the grid-independent Leray solution is also expressed clearly in figure 7(a). A nearly grid-independent solution is obtained at $N=64$ for $\Delta \lesssim \ell / 16$. 
(a)

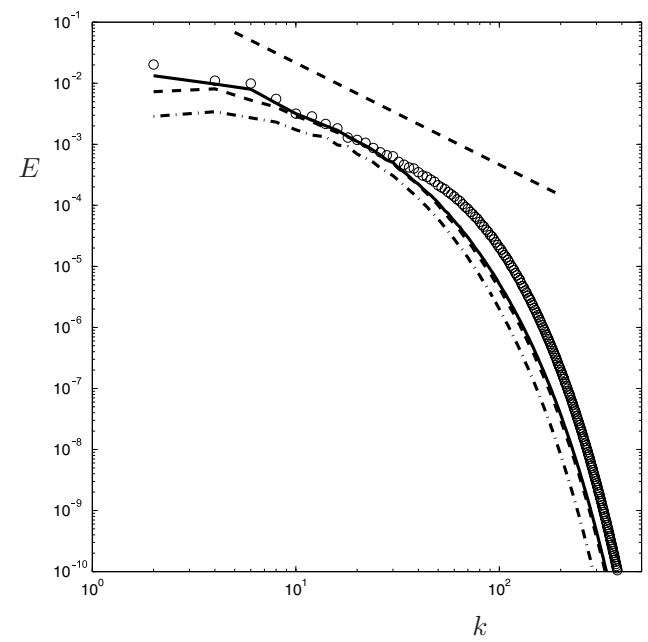

(b)

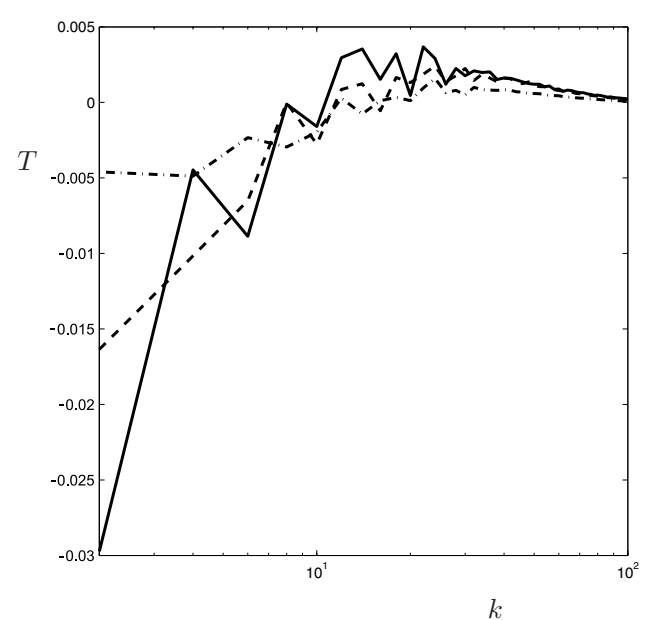

(c)

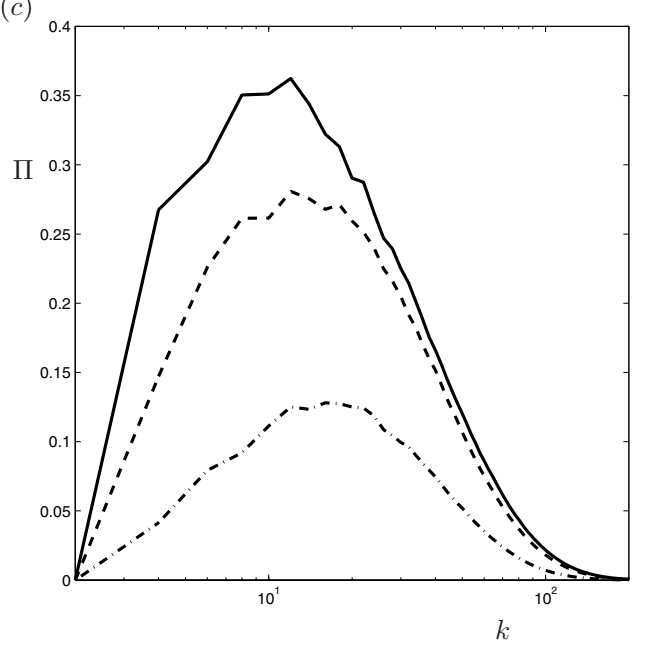

Figure 8. The kinetic energy spectrum $E(a)$, the energy-transfer spectrum $T(b)$ and the power spectrum $\Pi(c)$ at time $t=0(0), t=0.25$ (solid), $t=0.5$ (dash) and $t=1$ (dash-dot) using a resolution $N^{3}$ with $N=512$.

Under-resolution as arises at $N=32$ is particularly clear in the initial stages, and at small filter widths. The results for the Leray model at $\Delta \approx \ell / 32$ provide a point of reference for assessing the influence of spatial discretization errors in the other sub-filter models. This is shown in figure $7(b)$. Compared to the filtered DNS results, the Leray model provides the most accurate results, and the lowest degree of sensitivity to under-resolution. The influence of under-resolution is particularly strong for the NS- $\alpha$ and modified Leray models, as can be seen from the results at $N=32$.

In a pragmatic approach to LES [52], a direct minimization of the combination of sub-filter modeling errors and spatial discretization errors was considered. In this way the deviation from the filtered DNS findings could be minimized while keeping the computational costs low. This approach yields an optimal filter width as a function of the spatial resolution, corresponding 
(a)

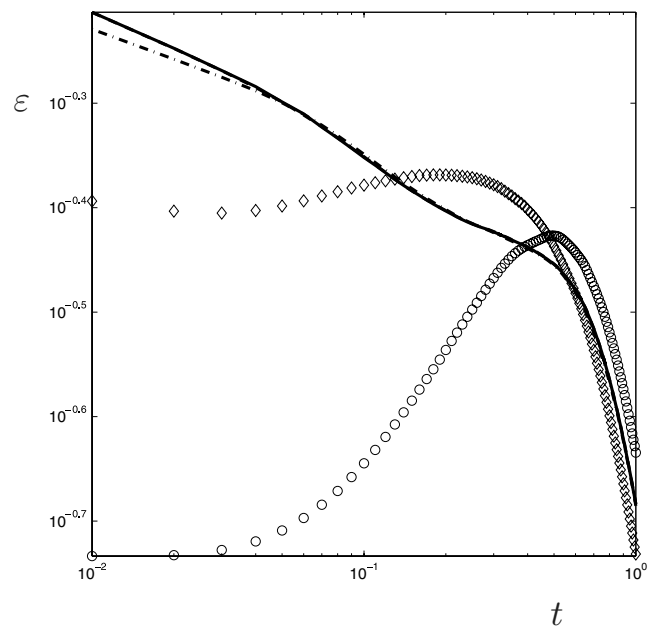

(b)

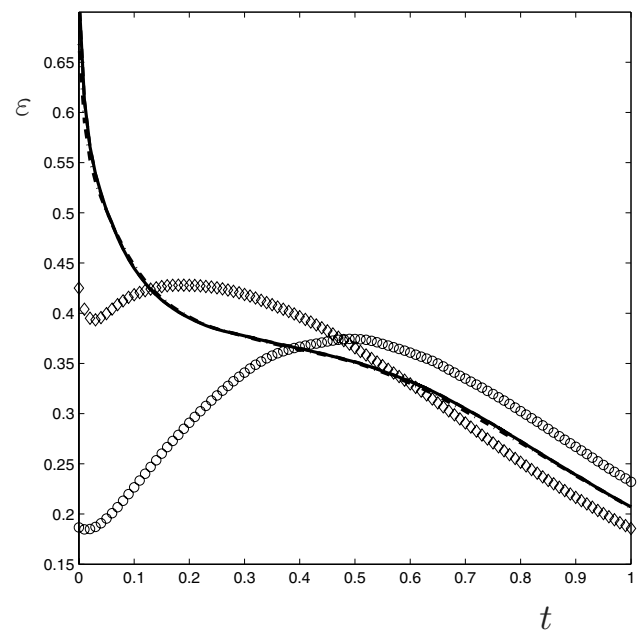

Figure 9. Evolution of the total dissipation rate $\varepsilon$ at resolutions $N^{3}$ with $N=32$ (०), $N=64$ $(\diamond), N=128$ (dash-dot), $N=256$ (dash) and $N=512$ (solid). In (a) we show $\varepsilon$ versus $t$ on a doubly-logarithmic plot and in $(b)$ a linear plot of $\varepsilon$ versus $t$ is shown.

to a lower overall simulation error. A drawback of this approach is that it requires detailed reference results to be available. Moreover, the theoretical basis for this error-minimization is unclear and the optimized simulation parameters are likely to be specific to the selected flow conditions. Therefore, we will not pursue this here but investigate in more detail the kinetic energy dynamics of the regularization models in the following section, to establish the observed error behavior more clearly.

\section{Regularized energy dynamics}

In this section, we analyze the energy dynamics in the large-eddy simulations by considering the energy spectrum $E$, the energy-transfer spectrum $T$ and the power spectrum $\Pi$. This yields an understanding of the spectral range of scales at which the dominant simulation errors occur in the regularization models. We will also consider the dissipation rate $\epsilon$, which is central to the energy decay. The energy spectra for the NS- $\alpha$ model were studied earlier in [20, 25], while spectra for the Leray model were presented in [12-14]. In two spatial dimensions this problem was considered in [47, 53].

We first consider the reference DNS results and subsequently discuss the LES findings.

\subsection{DNS predictions of spectral energy dynamics}

In figure 8 we collected $E, T$ and $\Pi$ at three instants of time. We note that the energy spectrum in (a) shows a region with approximately $k^{-5 / 3}$ behavior [29, 30], which is seen to decay selfsimilarly. The energy-transfer spectrum in $(b)$ shows a region of negative values at low $k$, implying a strong flow of energy toward smaller scales. The somewhat irregular dependence of $T$ on $k$ for small $k$, seen at $t=0.25$ is due to the uncorrelated initial condition that was adopted. At later times the $k$-dependence of the transfer spectrum is more regular, reflecting the developed correlations in the turbulent flow. The power spectrum in $(c)$ provides a further 
(a)

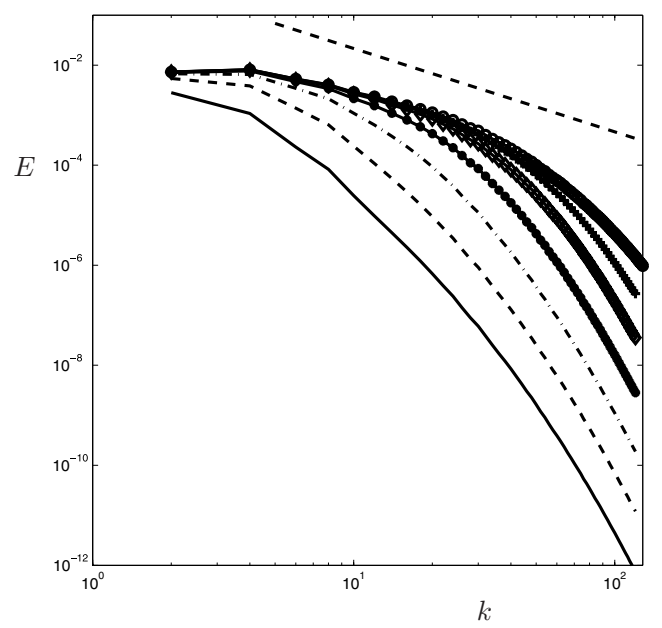

(b)

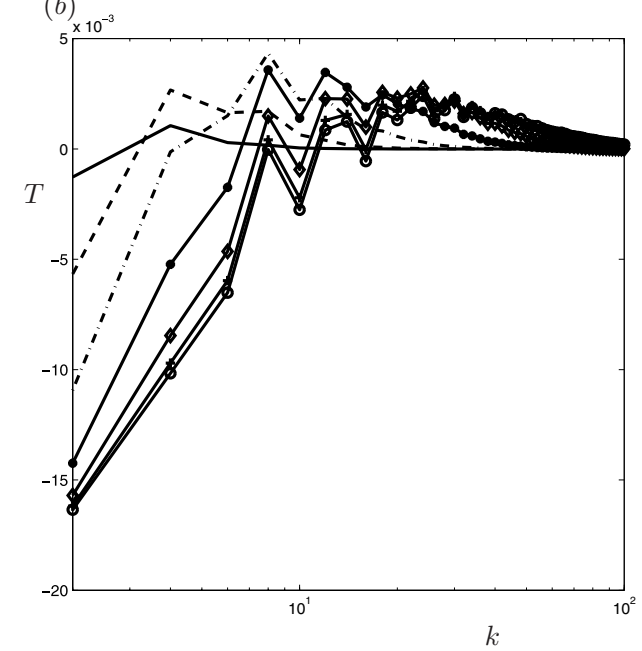

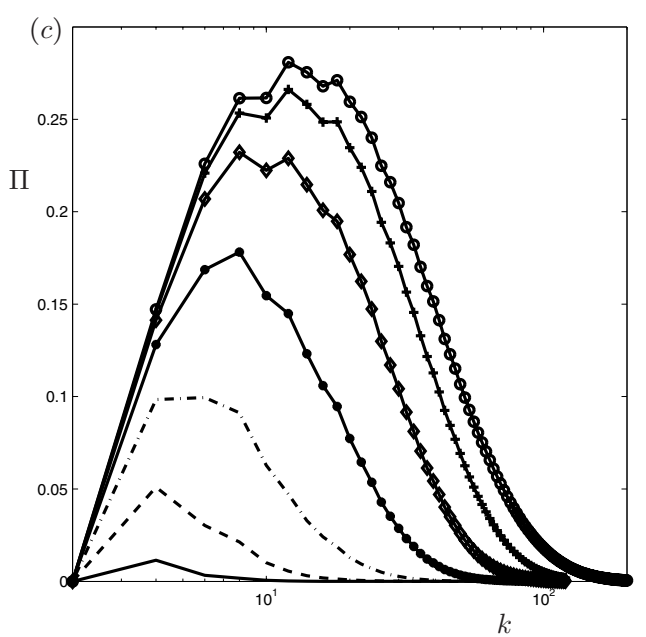

Figure 10. The kinetic energy spectrum $E(a)$, the energy-transfer spectrum $T(b)$ and the power spectrum $\Pi(c)$ at time $t=0.5$ quantifying the effect of Helmholtz filtering the DNS data obtained at resolution $N^{3}$ with $N=512$. The filtered DNS data were obtained at $\alpha=\ell / 10$ (solid), $\ell / 20$ (dash), $\ell / 40$ (dash-dot), $\ell / 80(*), \ell / 160(\diamond), \ell / 320(+)$ and $0(\circ)$, and subsequently injected onto a grid of $N^{3}$ with $N=128$ after which the spectral quantity was evaluated.

characterization of the energy transfer in the flow. As time progresses, the wavenumber at which $\Pi$ is maximal tends toward larger $k$ values, i.e., smaller length-scales. At $t=0.5$ there appears a short range of wavenumbers at which $\Pi$ is approximately flat, indicating the extent of the inertial range in this case.

The dissipation rate $\epsilon$ is given by

$$
\epsilon(t)=\int_{0}^{k_{\max }} \frac{2 k^{2}}{R e} E(k, t) \mathrm{d} k .
$$

It is dominated by the smaller scales in the flow and will depend sensitively on the specific regularization model that is adopted. In figure 9 we show the convergence of the dissipation 

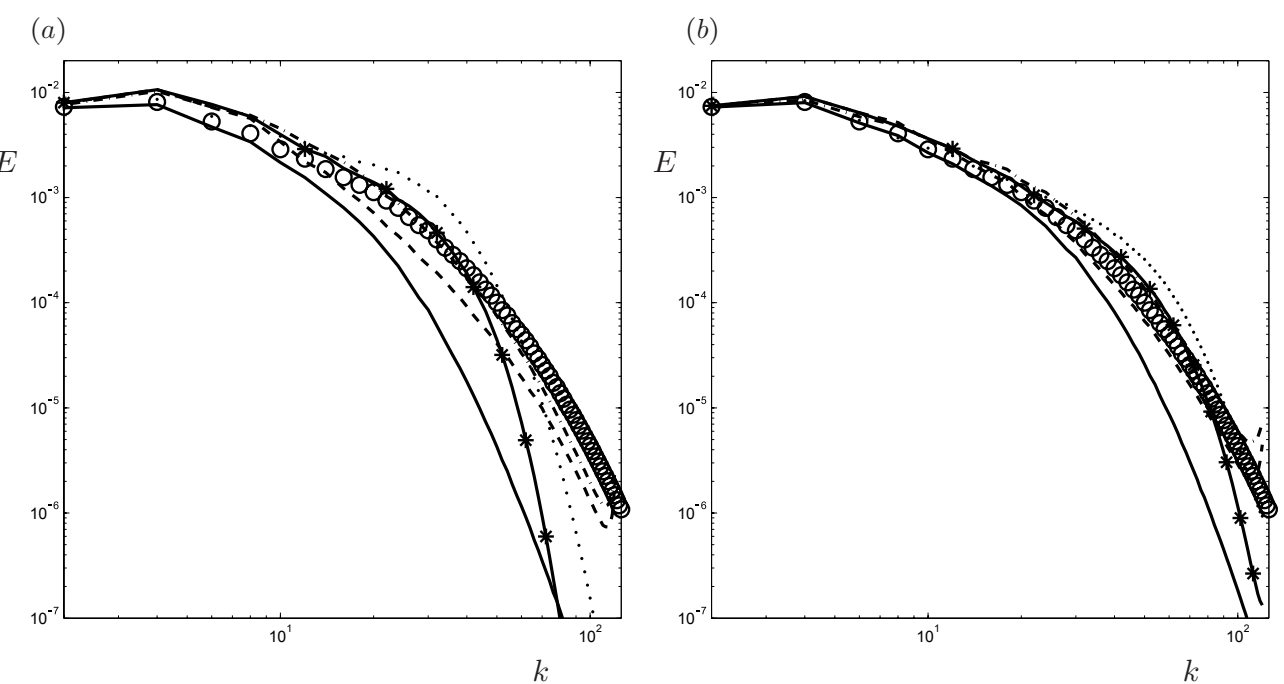

Figure 11. The spectrum of kinetic energy at $t=0.5$ and $\alpha=\ell / 80(a)$ and $\alpha=\ell / 160(b)$. The unfiltered DNS spectrum is marked by $\circ$, filtered DNS spectrum (solid) and LES predictions with Leray (dash), NS- $\alpha$ (dash-dot), modified Leray (dot) and simplified Bardina (*) at spatial resolution $N=128$.

rate as a function of the spatial resolution. In these simulations under-resolved DNS with $N \in\{32,64,128,256\}$ were computed. We note an underestimation of $\epsilon$ during the initial stages of development, in case $N=32$ or 64 , but near grid-independence is established for $N \geqslant 128$. During the initial stages of the evolution the grid-independent dissipation-rate is seen to decrease rapidly. This is followed by a short period of slower decrease of $\varepsilon$, roughly between $t=0.2$ and $t=0.5$, which from $t \approx 0.5$ onward displays a second regime of slightly more rapid decrease in which $\varepsilon$ depends approximately linearly on $t$. These stages are well appreciated by comparing both the logarithmic and the linear plots of the data in figure 9 . The last stage in the developing flow is quite well captured even at the coarser resolutions.

The effect of explicit Helmholtz filtering on the spectra $(E, T, \Pi)$ is summarized in figure 10. We observe a gradual reduction of the energy spectrum with an increasing filter width. As expected, this reduction is strongest for the smallest scales. Corresponding to the filtering the energy transfer is less pronounced and thus, also the amplitude of the power spectrum becomes smaller with an increasing filter width. This also leads to a shift to smaller $k$ of the location of maximal $\Pi$, i.e., fewer 'donor' modes transfer energy to a range of small scales whose size increases with increasing $\Delta$. We also considered the effect of filtering on the dissipation rate. An increased filter width was found to strongly reduce $\epsilon$ and to slightly reduce the time at which the first regime of algebraic decay transitions into the second stage of more rapid decay. These reference data will next be used to assess the quality of the regularization models.

\subsection{LES predictions of energy dynamics}

The predictions of $(E, T, \Pi)$ as obtained with the regularization models will be presented next. The energy spectra are shown in figure 11 . Both at $\alpha=\ell / 80$ and $\alpha=\ell / 160$ all regularization models predict spectra whose high- $k$ tails are considerably above that of the filtered DNS. In fact, the observed spectral tails are quite close to unfilterd DNS spectra. The Leray prediction 
(a)

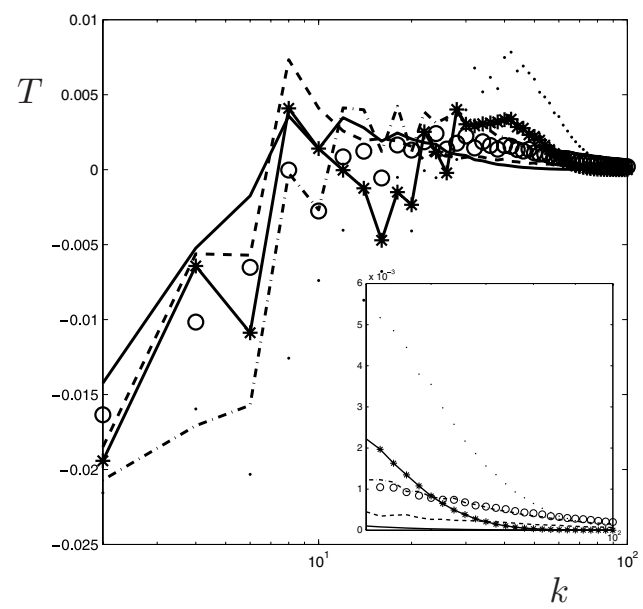

(c)

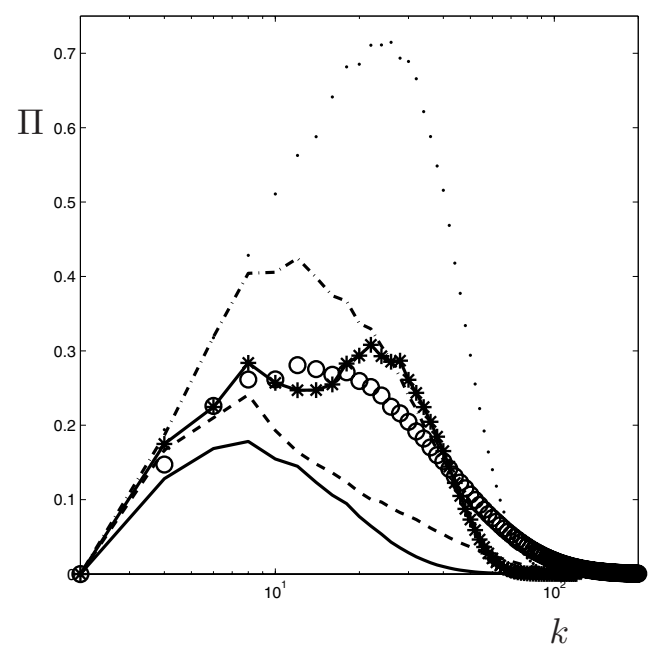

(b)

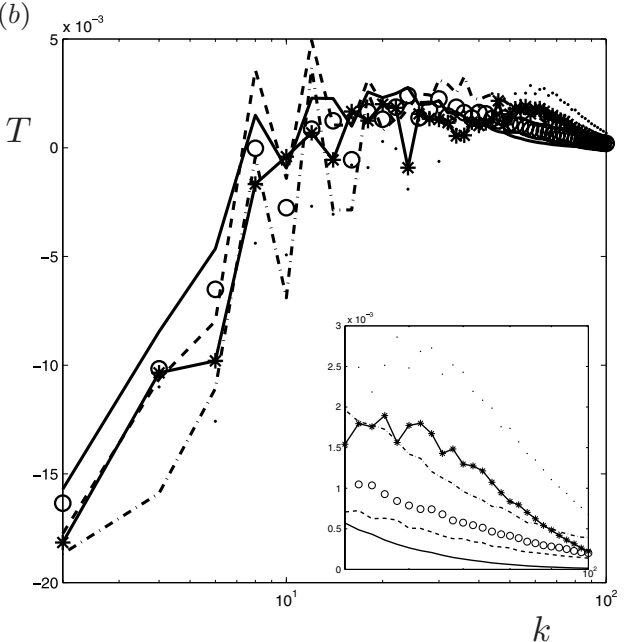

$(d)$

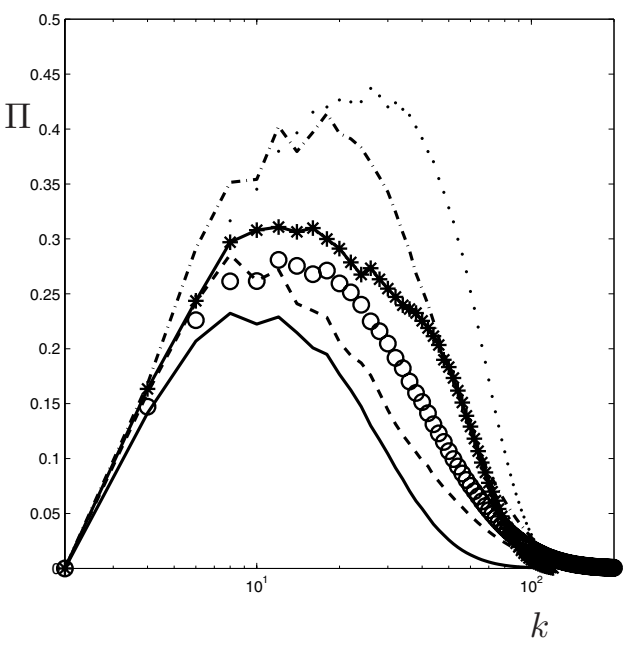

Figure 12. Energy-transfer spectrum at $t=0.5$ with $\alpha=\ell / 80(a)$ and $\alpha=\ell / 160(b)$ comparing the unfiltered DNS data (o) with the filtered DNS data (solid), and LES predictions with Leray (dash), NS- $\alpha$ (dash-dot), modified Leray (dot) and simplified Bardina (*). In the insets the tail of the spectrum is enlarged. Energy power spectrum at $t=0.5$ with $\alpha=\ell / 80$ (c) and $\alpha=\ell / 160$ (d). A spatial resolution $N=128$ was used.

is generally closest to the filtered DNS spectrum over a wide range of wavenumbers. In contrast the modified Leray model yields a considerable overprediction, even compared to unfiltered DNS, at a range of intermediate scales. After this 'spectral bottleneck' the modified Leray model displays a much stronger decay of the tail of the spectrum. This very strong reduction of the tail of the spectrum is also observed in the simplified Bardina model, while the NS- $\alpha$ spectrum is generally similar in shape to the Leray model, albeit with more energy in the smaller scales.

The regularization models' results for the energy-transfer spectrum and the power spectrum are collected in figure 12. The energy-transfer spectrum shows a complex dependence on $k$. For the smaller as well as the larger $k$ values, the Leray model is seen 
(a)

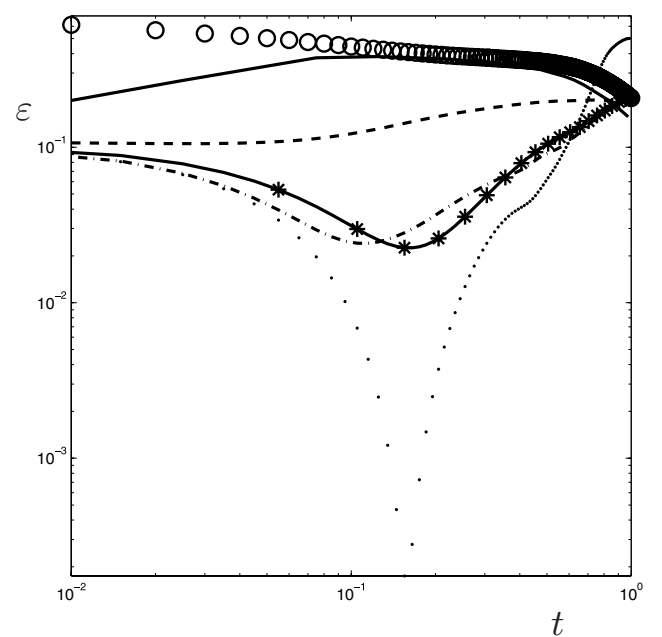

(b)

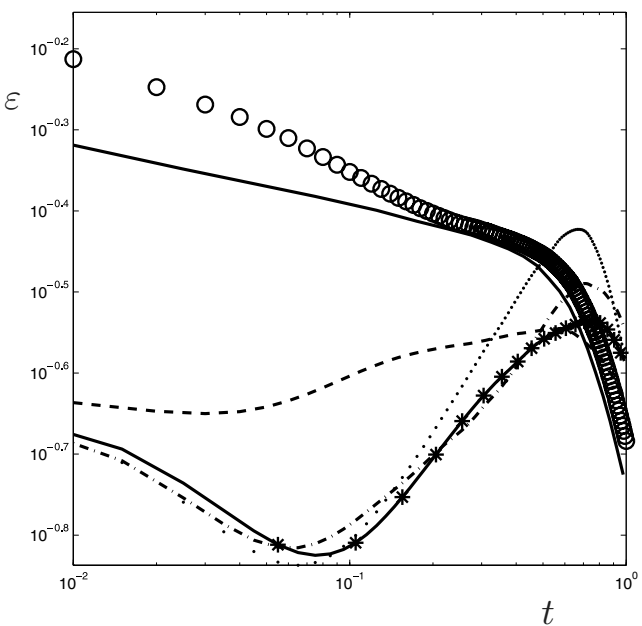

Figure 13. Evolution of the dissipation rate $\varepsilon$ with $\alpha=\ell / 80$ (a) and $\alpha=\ell / 160$ (b) comparing the unfiltered DNS data ( $\circ$ ) with the filtered DNS data (solid), and LES predictions with Leray (dash), NS- $\alpha$ (dash-dot), modified Leray (dot) and simplified Bardina (*) at spatial resolution $N=128$.

to compare quite well with the filtered DNS data. In contrast, the modified Leray model is seen to generate too strong transfer of energy from the largest to the smaller scales and a corresponding overestimate of $T$ at the smaller scales. The simplified Bardina model displays considerable deviations from the filtered DNS results in an intermediate range of scales and a similar overprediction of $T$ for the larger scales. As may be seen from the inset, at very large $k$ the simplified Bardina model does appear to follow the general behavior of $T$. This is not the case for the NS- $\alpha$ model, which shows levels of the transfer spectrum comparable to or even larger than arise in the unfiltered DNS. This behavior is more cleanly expressed by the power spectrum. Here, it is much more clear that the Leray model is closest to the filtered DNS results, both at $\alpha=\ell / 160$ and $\alpha=\ell / 80$. The other regularization models display too strong overall energy transfer to smaller scales, even stronger than in the unfiltered DNS, in most cases. This is particularly pronounced for the modified Leray model, and underlines the observed fragmented impression of flow structures in snapshots of the numerical solution (cf figure 1). The overprediction of $\Pi$ due to the NS- $\alpha$ is also readily observed and correlates well with the observed overestimated variability of the small scales in figure 1.

Finally, we consider the dissipation rate. In figure 13, we compare the observed dissipation rates in the regularization models with the filtered and unfiltered DNS results. The considerable underestimation of the dissipation rate by each of these sub-filter models is readily appreciated. The Leray model is seen to provide the highest levels of dissipation, but nevertheless much too low and with a time-dependence that does not reflect the DNS findings well. The other regularization models yield even lower levels of dissipation during most of the flow development. Moreover, these models first show a minimal $\varepsilon$ at $t \approx 1 / 10$ after which the dissipation rate increases. At $\alpha=\ell / 80$ we note that the modified Leray model gives rise to particularly low dissipation rates around $t=0.15$, which reflects the very fragmented flow-structuring seen in figure 1 . The fact that both the shape as a function of time and the level of $\varepsilon$ are incorrectly represented by all regularization models is at the heart of the (slight) overestimation of the small-scale variability. This motivates further developments of regularization models in the future. 
(a)

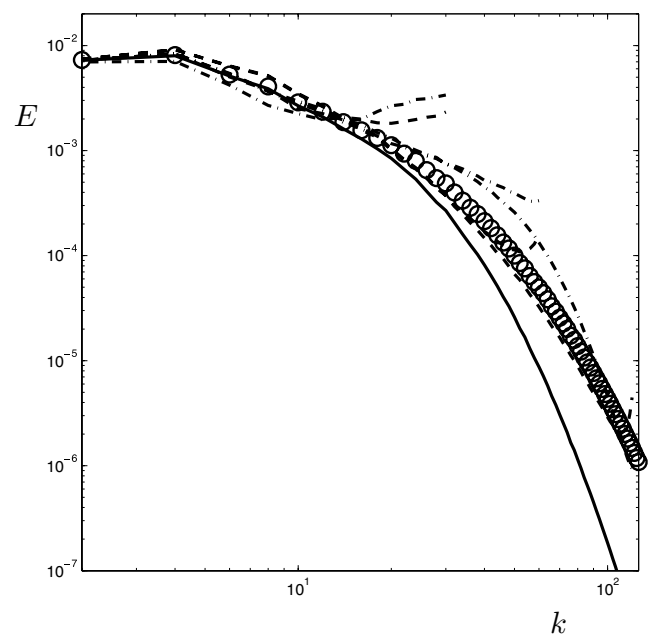

(b)

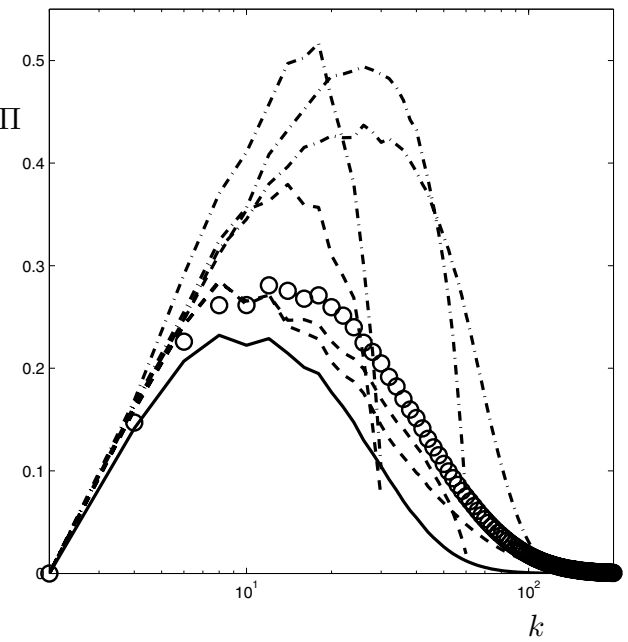

Figure 14. The spectrum of kinetic energy $(a)$ and the energy power spectrum $(b)$ at $t=0.5$ and $\alpha=\ell / 160$ comparing the unfiltered DNS data (o) with the filtered DNS data (solid), and LES predictions with Leray (dash) and modified Leray (dashdot) at spatial resolutions $N=32$, 64 and 128.

All findings presented in this subsection were obtained at a high spatial resolution of $N=128$. As observed before, an under-resolution of the small scales can lead to additional numerical effects. These are illustrated for the energy spectrum and the power spectrum in figure 14. Indeed, we observe considerable deviations in the results when the resolution is too low. However, the final conclusions regarding the suitability of the Leray and the modified Leray models can be inferred even on the basis of the coarser grids. The Leray predictions are quite close to the DNS findings while the modified Leray model induces far too many small scales in the solution. Under-resolution at $N=32$ displays an overestimated $\Pi$ for both models. An increased resolution does produce a clear convergence for Leray and slightly less satisfactory for the modified Leray model.

\section{Concluding remarks}

In this paper, we studied the use of regularization models for the large-eddy prediction of homogeneous decaying isotropic turbulence. This flow was treated on the basis of the pseudospectral discretization method, at flow conditions that allow a well-resolved direct numerical simulation at $O\left(10^{8}\right)$ grid points. Hence, a clear point of reference for assessing the various regularization models was obtained. However, this DNS option by itself is of little practical use in view of the required computational costs. The new coarsened flow description based on the direct regularization of the convective nonlinearities in the Navier-Stokes equations was considered and tested instead.

An elegant aspect of the regularization approach is that it provides a systematic method for deriving a closure for the coarsened flow description from an assumed dynamic principle $[11,12,17]$. In addition, rigorous mathematical results are available that establish existence, uniqueness and regularity of the solution to the modeled large-eddy equations. Four examples were included in this paper, i.e., the Leray model, the NS- $\alpha$ model, the modified Leray model 
and the simplified Bardina model. This study focused on the quality of these regularization principles as sub-filter models for turbulence. We distinguished between grid-independent solutions to the regularized equations, to assess the capturing of the primary turbulent flow features, and solutions obtained at marginal sub-filter resolution $\Delta / h$, to assess the accuracy of these models under more realistic LES resolution conditions.

The regularization models were compared against the filtered DNS results over a wide range of filter widths $\Delta$, using the Helmholtz filter. Each of these models was found to yield considerable errors in the predictions in the case of very large filter widths, i.e., in cases where $\Delta / \ell \gtrsim 1 / 4$, where $\ell$ is the computational box-size. Conversely, all regularization models were found to yield accurate predictions in cases with small filter widths, i.e., $\Delta / \ell \lesssim 1 / 64$. In these simulations, the contribution of the sub-filter model was very limited and the results for a variety of flow properties were quite similar to those found without the sub-filter model.

The main interest for regularization models is in the regime in which $1 / 32 \lesssim \Delta / \ell \lesssim 1 / 8$. In this range of filter widths, the contribution of the sub-filter model to the total flux is considerable as is the saving of computational effort. The detailed assessment of the regularization models discussed in this paper was based on the total energy, the Taylor Reynolds number and the velocity skewness. Generally speaking, the Leray model provides quite accurate approximations in this range of filter widths, compared to the filtered DNS findings. The modified Leray model was found to yield rather large errors, expressed by a too strong presence of the smaller scales in the modeled flow. The simplified Bardina and NS- $\alpha$ models we found yield results that were in most cases quite comparable to each other, but at a larger error than the Leray model. All models were found to underestimate the initial dissipation rate, leading to an overprediction of the resolved turbulent kinetic energy and the tail of the energy spectrum.

From these observations we may make a proposal for the maximal ratio between the filter width $\Delta$ and the Kolmogorov length-scale $\eta$ for which the grid-independent solutions to the regularization models give a good correlation with the filtered DNS solution. For the simulation with the initial Taylor Reynolds number $R e_{\lambda}=100$ we observed that $\eta / \ell$ evolves from an initial value of about $2 \times 10^{-3}$ to a value of about $3 \times 10^{-3}$ at $t=1$. From flow impressions as in figure 1 and the quantitative analysis in the previous two sections, we may infer that as long as $1 / 16 \lesssim \Delta / \ell \lesssim 1 / 8$ the general correspondence between the Leray and NS- $\alpha$ models on the one hand, and filtered DNS on the other hand, is quite acceptable. This implies ratios $20 \lesssim \Delta / \eta \lesssim 60$. For $\Delta / \eta \lesssim 20$ the quantitative agreement with filtered DNS is generally quite close for the Leray, $\mathrm{NS}-\alpha$ and simplified Bardina models. For the less restrictive bound $\Delta / \eta \lesssim 60$ the agreement is more qualitative, with the Leray model as the more accurate option.

These 'physical' resolution requirements also illustrate the potential gain in computing time that is achievable with regularization models. Adhering to an LES resolution of two grid-cells per $\Delta[50,56]$ and a DNS resolution of one grid-cell per $\eta$ [43-45], we may hence estimate

$$
\frac{N_{\mathrm{LES}}}{N_{\mathrm{DNS}}}=\frac{1 / h_{\mathrm{LES}}}{1 / h_{\mathrm{DNS}}}=2 \frac{\eta}{\Delta}
$$

which implies for the ratio of grid-points: $4 \times 10^{-5} \lesssim 8(\eta / \Delta)^{3} \lesssim 1 \times 10^{-3}$. The most conservative estimate, which also provides accurate predictions, hence implies a saving of a factor of 1000 in memory. Factoring in the increased time-step, the total computational effort scales as $\left(N_{\text {LES }} / N_{\text {DNS }}\right)^{4}[50,58]$, implying at least a factor of $10^{4}$ faster simulations. This estimate increases to a factor of $8 \times 10^{5}$ in the most progressive estimate. In actual simulations reported in this paper, computing times for a full DNS at a resolution of $512^{3}$ are on the order of $160 \mathrm{~h}$ on 16 CPUs. Given the measured speed-up of the code, this amounts to an equivalent of 
about 2400 h, i.e., 100 days, on a single CPU (corresponding to an SGI Altix 3700 machine). A large-eddy simulation employing a regularization model at a coarsening of $\Delta / \eta=20$, would imply an LES grid-spacing of $h_{\text {LES }}=10 h_{\text {DNS }}$, i.e., a resolution of about $50^{3}$. Since a simulation at a resolution of $32^{3}$ requires about $2 \mathrm{~min}$, and at $64^{3}$ approximately half an hour, this corresponds to a factor between 5000 and 75000 savings. Turning to the coarser option $\Delta / \eta=60$ we infer $h_{\mathrm{LES}}=30 h_{\mathrm{DNS}}$, i.e., a resolution of $17^{3}$, bringing the saving in the range 75000 to $1 \times 10^{6}$ at this instance. Hence, a huge saving in computational costs may be realized, without compromising the general turbulence structures and quantitative predictions too much.

With the grid-independent solution as the point of reference, it is of interest to investigate the extent with which the solution deteriorates if the resolution is decreased. In case the spatial resolution was lowered from $N=128$ to $N=64$ or even $N=32$ the quality of (some of) the predictions was considerably affected. In particular, the modified Leray model was shown to yield solutions with a strong overestimation of the smaller scales and consequently, less accurate representation of the energy dynamics and statistical flow properties. In contrast, the Leray model was found to yield the most acceptable sensitivity levels while the solution obtained with either the simplified Bardina and NS- $\alpha$ models yielded considerably deteriorated accuracy levels upon coarsening.

The mathematical elegance of regularization models has important advantages in case consistent models of small-scale turbulence are developed. In particular, when turbulence is further complicated through interactions with other mechanisms, e.g., combustion, rotation, stratification or large ensembles of particles, it is essential that the sub-filter modeling is obtained from a systematic framework. Presently available regularization models such as the Leray and the NS- $\alpha$ models were shown to underestimate the decay of turbulent kinetic energy in homogeneous decaying isotropic turbulence. Hence, it is still an open challenge to formulate a regularization principle from which a sub-filter model can be derived that represents the decay of energy in closer agreement with the filtered DNS findings. This is the subject of ongoing research.

\section{Acknowledgments}

The computations reported in this paper were made possible through a grant of the National Computing Foundation (NCF) (SH-061-07) in the Netherlands. AKK contributed to this work as part of his research grant in the FOM program 'Turbulence and its role in energy conversion processes'. The work of E.S.T. was supported in part by the NSF grant no DMS-0504619, the BSF grant no 2004271 and the ISF grant no 120/06.

\section{References}

[1] Pope S B 2000 Turbulent Flows (Cambridge: Cambridge University Press)

[2] Lesieur M 1990 Turbulence in Fluids (Dordrecht: Kluwer)

[3] McComb W D 1990 The Physics of Fluid Turbulence (Oxford: Oxford University Press)

[4] Geurts B J 2004 Elements of Direct and Large-Eddy Simulation (R T Edwards)

[5] Sagaut P 2001 Large Eddy Simulation for Incompressible Flows: An Introduction (Scientific Computation) (Berlin: Springer)

[6] Germano M 1992 Turbulence: the filtering approach J. Fluid Mech. 238325

[7] Smagorinsky J 1963 General circulation experiments with the primitive equations Mon. Weather Rev. 9199

[8] Bardina J, Ferziger J and Reynolds W 1980 Improved subgrid scale models for large eddy simulation Am. Inst. Aeronaut. Astronaut. Paper 80 80-1357 
[9] Bardina J, Ferziger J H and Reynolds W C 1984 Improved turbulence models based on LES of homogeneous incompressible turbulent flows Dept. Mech. Eng. Report No. TF-19, Stanford

[10] Vreman A W, Geurts B J and Kuerten J G M 1994 Realizability conditions for the turbulent stress tensor in large eddy simulation J. Fluid Mech. 278351

[11] Leray J 1934 Sur les movements d'un fluide visqueux remplaissant l'espace Acta Math. 63193

[12] Geurts B J and Holm D D 2003 Regularization modelling for large eddy simulation Phys. Fluids 15 L13

[13] Geurts B J and Holm D D 2006 Leray and NS- $\alpha$ modeling of turbulent mixing J. Turbulence 7 1-33

[14] Cheskidov A, Holm D D, Olson E and Titi E S 2005 On a Leray- $\alpha$ model of turbulence Phil. Trans. R. Soc. A 461 629-49

[15] Chen S, Foias C, Holm D D, Olson E, Titi E S and Wynne S 1999 A connection between the Camassa-Holm equations and turbulent flows in channels and pipes Phys. Fluids 11 2343-53

[16] Chen S, Foias C, Holm D D, Olson E, Titi E S and Wynne S 1998 Camassa-Holm equations as a closure model for turbulent channel and pipe flow Phys. Rev. Lett. 81 5338-41

[17] Foias C, Holm D D and Titi E S 2001 The Navier-Stokes-alpha model of fluid turbulence Physica D 152505

[18] Foias C, Holm D D and Titi E S 2002 The three dimensional viscous Camassa-Holm equations, and their relation to the Navier-Stokes equations and turbulence theory J. Dyn. Diff. Eqns 14 1-35

[19] Chen S, Foias C, Holm D D, Olson E, Titi E S and Wynne S 1999 The Camassa-Holm equations and turbulence Physica D 133 49-65

[20] Mohseni K, Kosović B, Shkoller S and Marsden J 2003 Numerical simulations of the Lagrangian averaged Navier-Stokes equations for homogeneous isotropic turbulence Phys. Fluids 15 524-44

[21] Cao Y, Lunasin E and Titi E S 2006 Global well-posedness of the three-dimensional viscous and inviscid simplified Bardina turbulence models Commun. Math. Sci. 4 823-48

[22] Layton W and Lewandowski R 2006 On a well-posed turbulence model Dicrete Continuous Dyn. Syst. B 6 111-28

[23] Berselli L C, Illiescu T and Layton W J 2006 Mathematics of Large-eddy Simulation of Turbulent Flows (Berlin: Springer) ISBN 3-540-26316-0

[24] Ilyin A, Lunasin E and Titi E S 2006 A modified-Leray- $\alpha$ subgrid scale model of turbulence Nonlinearity 19 879-97

[25] Chen S, Holm D D, Margolin L G and Zhang R 1999 Direct numerical simulations of the Navier-Stokes alpha model Physica D 133 66-83

[26] Cao C, Holm D and Titi E S 2005 On the Clark- $\alpha$ model of turbulence: global regularity and long-time dynamics J. Turbulence 6 1-11

[27] Stein E M 1971 Singular Integrals and Differentiability of Functions (Princeton, NJ: Princeton University Press) ISBN13: 978-0-691-08079-6

[28] Geurts B J and Holm D D 2002 Alpha-modeling strategy for LES of turbulent mixing Turbulent Flow Computation-Fluid Mechanics and Its Applications vol 66, ed D Drikakis and B J Geurts (Dordrecht: Kluwer) pp 237-78

[29] Kolmogorov A N 1991 The local structure of turbulence in an incompressible fluid with very large Reynolds numbers Proc. R. Soc. 4341890

Kolmogorov A N 1941 The local structure of turbulence in an incompressible fluid with very large Reynolds numbers Dokl. Akad. Nauk SSSR 30301 (first published)

[30] Frisch U 1995 Turbulence: The Legacy of A.N. Kolmogorov (Cambridge: Cambridge University Press)

[31] Vreman A W, Geurts B J and Kuerten J G M 1997 Large-eddy simulation of the turbulent mixing layer J. Fluid Mech. 339357

[32] Geurts B J 2006 Interacting errors in large-eddy simulation: a review of recent developments J. Turbulence 179596 (JOT-2005-0106.R1)

[33] Bos, van der F and Geurts B J 2005 Commutator-errors in the filtering approach to large-eddy simulation Phys. Fluids 17035108

[34] Stolz S and Adams N A 1999 An approximate deconvolution procedure for large-eddy simulation Phys. Fluids 111699

[35] Germano M, Piomelli U, Moin P and Cabot W H 1991 A dynamic subgrid-scale eddy viscosity model Phys. Fluids 31760

[36] Kuerten J G M, Geurts B J, Vreman A W and Germano M 1999 Dynamic inverse modeling and its testing in large-eddy simulations of the mixing layer Phys. Fluids 113778

[37] Geurts B J 1997 Inverse modeling for large-eddy simulation Phys. Fluids 93585

[38] Eyink G L 2005 Locality of turbulent cascades Physica D 20791

[39] Chen S, Eyink G L, Xiao Z and Wan M 2006 Is the Kelvin theorem valid for high-reynolds-number turbulence? Phys. Rev. Lett. 97144505 
[40] Holm D D 1999 Fluctuation effects on 3D Lagrangian mean and Eulerian mean fluid motion Physica D 133215

[41] Holm D D, Marsden J E and Ratiu T S 1998 Euler-Poincaré models of ideal fluids with nonlinear dispersion Phys. Rev. Lett. 804173

[42] Winckelmans G S, Jeanmart H, Wray A A, Carati D and Geurts B J 2001 Tensor-diffusivity mixed model: balancing reconstruction and truncation Modern Simulation Strategies for Turbulent Flow ed B J Geurts (Philadelphia, PA: R T Edwards) p 85

[43] Kuczaj A K and Geurts B J 2007 Mixing in manipulated turbulence J. Turbulence 7 1-28 DOI: $10.1080 / 14685240600827534$

[44] Young A J 1999 Investigation of renormalization group methods for the numerical simulation of isotropic turbulence Thesis University of Edinburgh

[45] Canuto C, Hussaini M Y, Quarteroni A and Zang T A 1988 Spectral Methods in Fluid Dynamics (Berlin: Springer)

[46] Kuczaj A K, Geurts B J and McComb W D 2006 Nonlocal modulation of the energy cascade in broadband-forced turbulence Phys. Rev. E 74016306

[47] Lunasin E, Kurien S, Taylor M A and Titi E S 2007 A study of the Navier-Stokes- $\alpha$ model for two-dimensional turbulence J. Turbulence 8 1-21

[48] Kuczaj A K, Geurts B J and Lohse D 2006 Response maxima in time-modulated turbulence: direct numerical simulations Europhys. Lett. 73 851-7 doi: 10.1209/epl/i2005-10486-2

[49] Meyers J, Geurts B J and Baelmans M 2003 Database-analysis of errors in large-eddy simulations Phys. Fluids 152740

[50] Geurts B J and Fröhlich J 2002 A framework for predicting accuracy limitations in large-eddy simulation Phys. Fluids 14 L41

[51] Meyers J, Geurts B J and Baelmans M 2005 Optimality of the dynamic procedure for large-eddy simulation Phys. Fluids 17045108

[52] Geurts B J and Meyers J 2006 Successive inverse polynomial interpolation to optimize Smagorinsky's model for large-eddy simulation of homogeneous turbulence Phys. Fluids $\mathbf{1 8} 118102$

[53] Lunasin E, Kurien S and Titi E S 2007 Spectral scaling of the Leray- $\alpha$ model for two-dimensional turbulence Preprint $0711.2829 \mathrm{v} 1$

[54] Klein M 2005 An attempt to assess the quality of large-eddy simulations in the context of implicit filtering Flow Turbulence Combustion 75 131-1147

[55] Celik I, Klein M, Freitag M and Janicka J 2006 Assessment measures for URANS/DES/LES: an overview with applications $J$. Turbulence 7 1-27

[56] Vreman A W, Geurts B J and Kuerten J G M 1994 Discretization error dominance over subgrid-terms in large eddy simulations of compressible shear layers Commun. Numer. Methods Eng. Math. 10785

[57] Vreman A W, Geurts B J and Kuerten J G M 1996 Comparison of numerical schemes in large eddy simulation of the temporal mixing layer Int. J. Numer. Methods Fluids 22297

[58] Geurts B J and Fröhlich J 2001 Numerical effects contaminating LES; a mixed story Modern Strategies for Turbulent Flow Simulation ed B J Geurts (Edwards Publishing) p 317 


\section{Endnotes}

(1) Author: Please check whether the edits made in the sentence 'An important class... model' retain your original intended sense.

(2) Author: Please check whether the edit made in the sentence 'The simplified Bardina... Leray model' retains your original intended sense.

(3) Author: Please provide publication details for references [4] and [58].

(4) Author: Please be aware that the colour figures in this article will only appear in colour in the Web version. If you require colour in the printed journal and have not previously arranged it, please contact the Production Editor now.

\section{Reference linking to the original articles}

References with a volume and page number in blue have a clickable link to the original article created from data deposited by its publisher at CrossRef. Any anomalously unlinked references should be checked for accuracy. Pale purple is used for links to e-prints at arXiv. 